\title{
Radical Deoxygenation of Hydroxyl Groups via Phosphites
}

\author{
Liming Zhang $^{\dagger}$ and Masato Koreeda ${ }^{\dagger \uparrow *}$ \\ Departments of Chemistry ${ }^{\top}$ and Medicinal Chemistry, ${ }^{\dagger}$ University of \\ Michigan, Ann Arbor, Michigan 48109-1055
}

Supporting Information

${ }^{1} \mathrm{H},{ }^{13} \mathrm{C}$ and ${ }^{31} \mathrm{P}$ NMR Spectra 
STANDARD 1H OBSERVE

Pulse Sequence: s2pul

Solvent: $\mathrm{CDC} 13$

Ambient temperature

File: phosphite-steroid

INOVA- 500 "Ni"

PULSE SEQUENCE

Pulse 41.4 degrees

Acq. time 2.666 .ec

Acq. time 2.666 o0c

Widh $3759.8 \mathrm{~Hz}$

32 repetitions 9649457 m

DATA PROCESSING

Dara proct

T size 32768

Total time $1 \mathrm{~min}, 25 \mathrm{sec}$

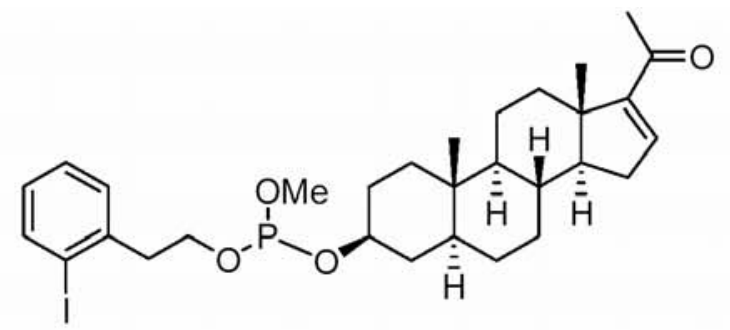

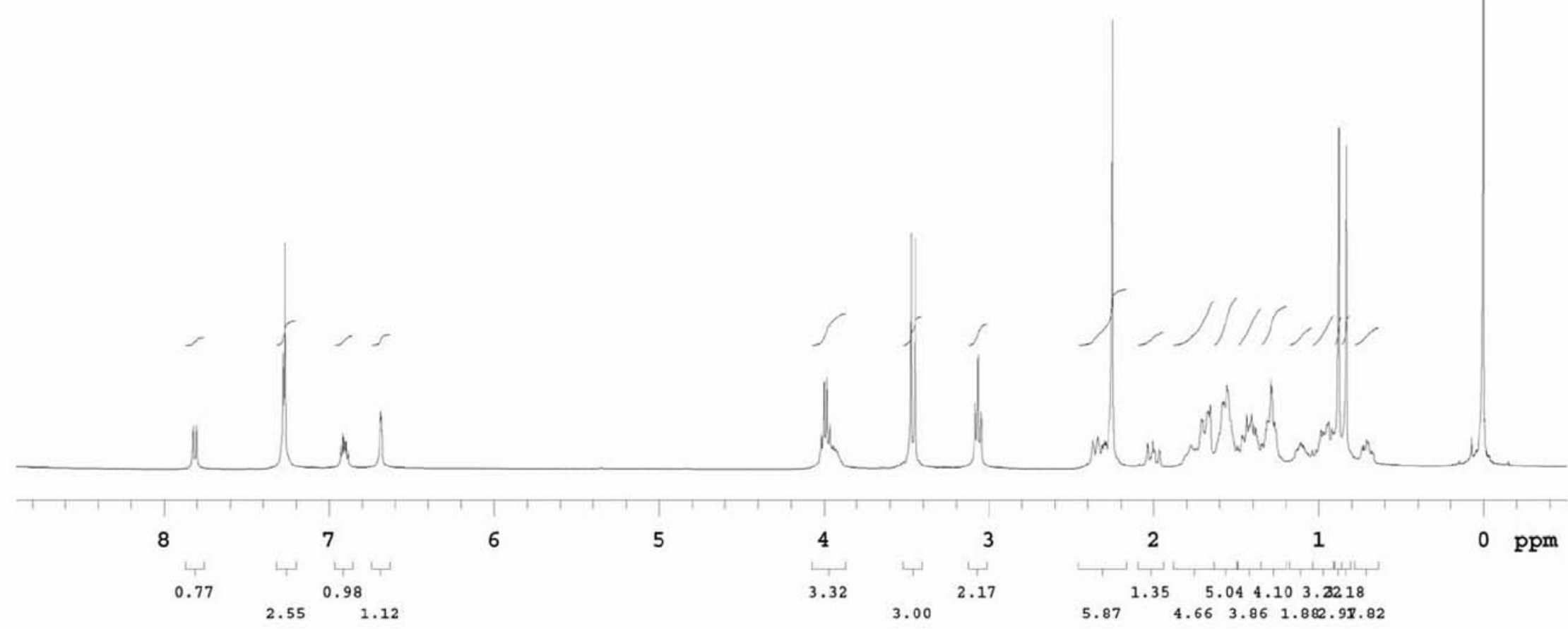


13C OBSERVE

Pulse Sequence: s2pul

Solvent: $\mathrm{CDC13}$

Ambient temperature

File: phosphite-steroid-c13

INOVA-500 "Ni"

PULSE SEQUENCE

Relax. delay $0.900 \mathrm{sec}$

Pulse 45.0 degrees

Acq. time $1.199 \mathrm{sec}$

width $25000.0 \mathrm{~Hz}$

368 repetitions

OBSERVE C13, $100.5712699 \mathrm{MHz}$ DECOUPLE H1, 399.9669644 MHz Power $44 \mathrm{~dB}$

continuously on

WALTZ-16 modulate

DATA PROCESSING

Line broadening $1.0 \mathrm{~Hz}$

T size 65536

Total time $70 \mathrm{hr}, 16 \mathrm{~min}, 25 \mathrm{sec}$

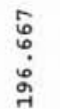

๙ั

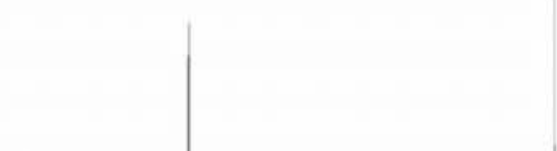

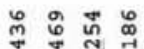

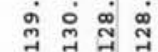

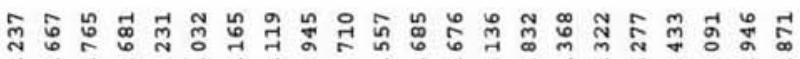

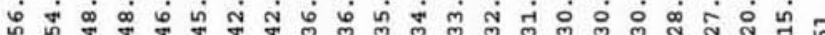

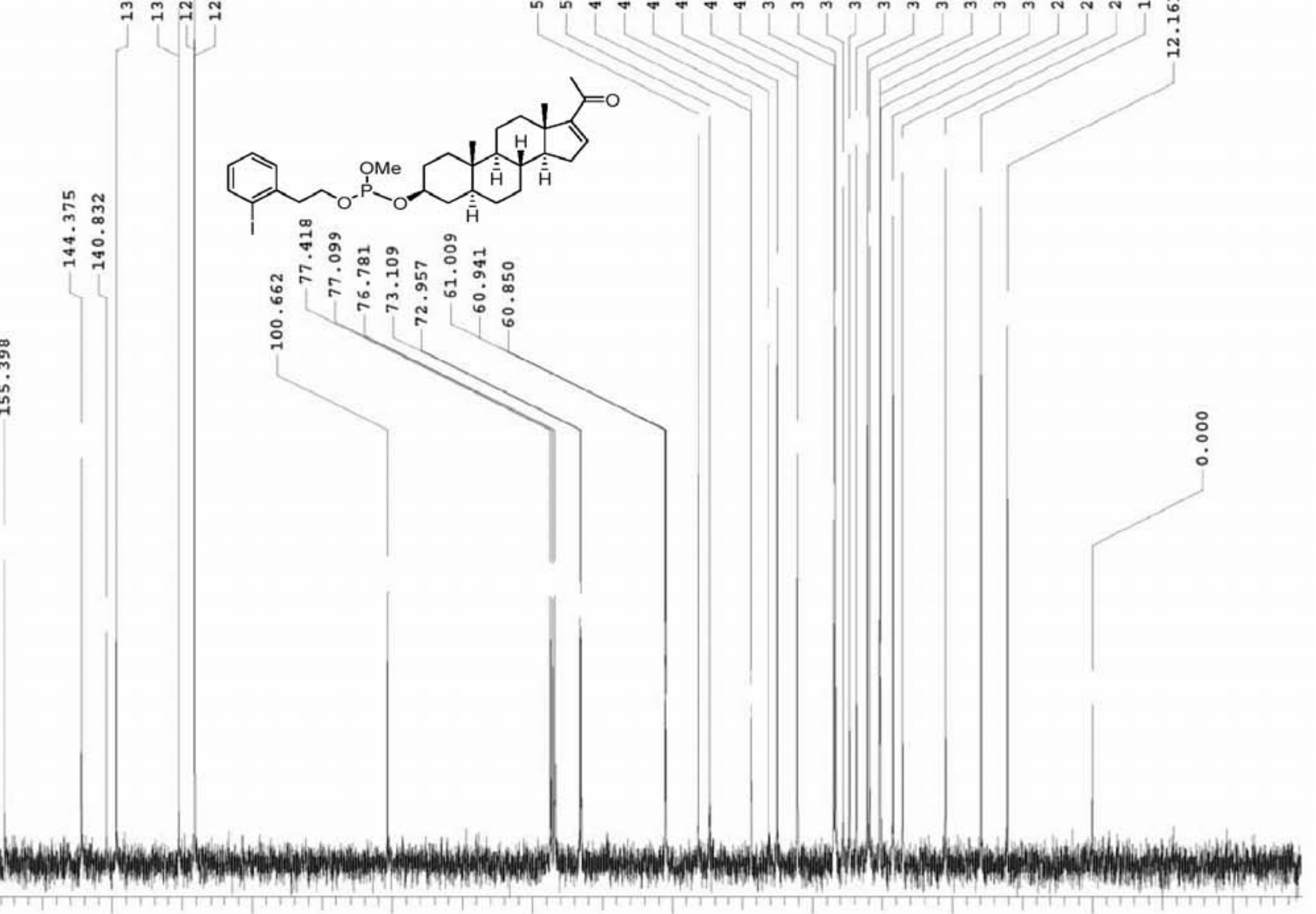

200

180

160

140

120

100

80

60

40

20

0

ppm 
PHOSPHORUS OBSERVE

STANDARD PARAMETERS

PHOSPHATE REGION

Pulse Sequence: s2pul

Solvent: $\mathrm{CDCl}_{3}$

Ambient temperature

File: phosphite-steroid-p31

INOVA-500 "Ni"

PULSE SEQUENCE

Pulse 45.0 degrees

Acq. time $0.800 \mathrm{sec}$

Width $60015.0 \mathrm{~Hz}$

128 repetitions

OBSERVE P31, $161.9086763 \mathrm{MH}$ DECOUPLE H1, $399.9669644 \mathrm{MHz}$ Power $44 \mathrm{~dB}$

continuously on

WALTZ-16 modulate

DATA PROCESSING

Line broadening $1.0 \mathrm{~Hz}$

T size 131072

Total time $1 \mathrm{~min}, 43 \mathrm{sec}$

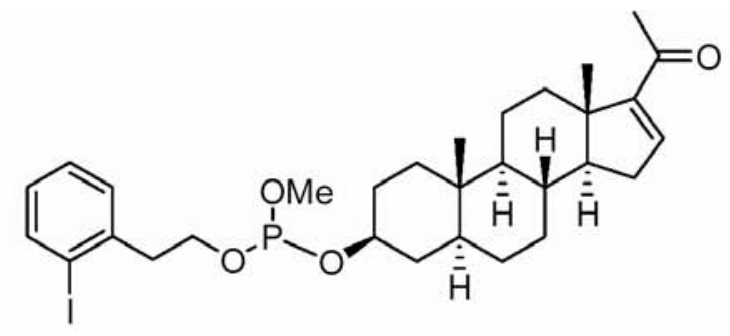


STANDARD 1H OBSERVE

Pulse Sequence: s2pul

Solvent: $\mathrm{CDCl} 13$

Temp. $25.0 \mathrm{C} / 298.1 \mathrm{~K}$

ile: phoophite-secondary-steroid-side-phosphonate

INOVA-500 "Ni"

PULSE SEQUENCE

Pulse 41.4 degrees

Acq. time $2.666 \mathrm{sec}$

width $6000.2 \mathrm{~Hz}$

60 repetitions

OBSERVE Hl, $399.9649469 \mathrm{MH}$

DATA PROCESSING

FT size 32768

Total time $5 \mathrm{~min}, 42 \mathrm{sec}$
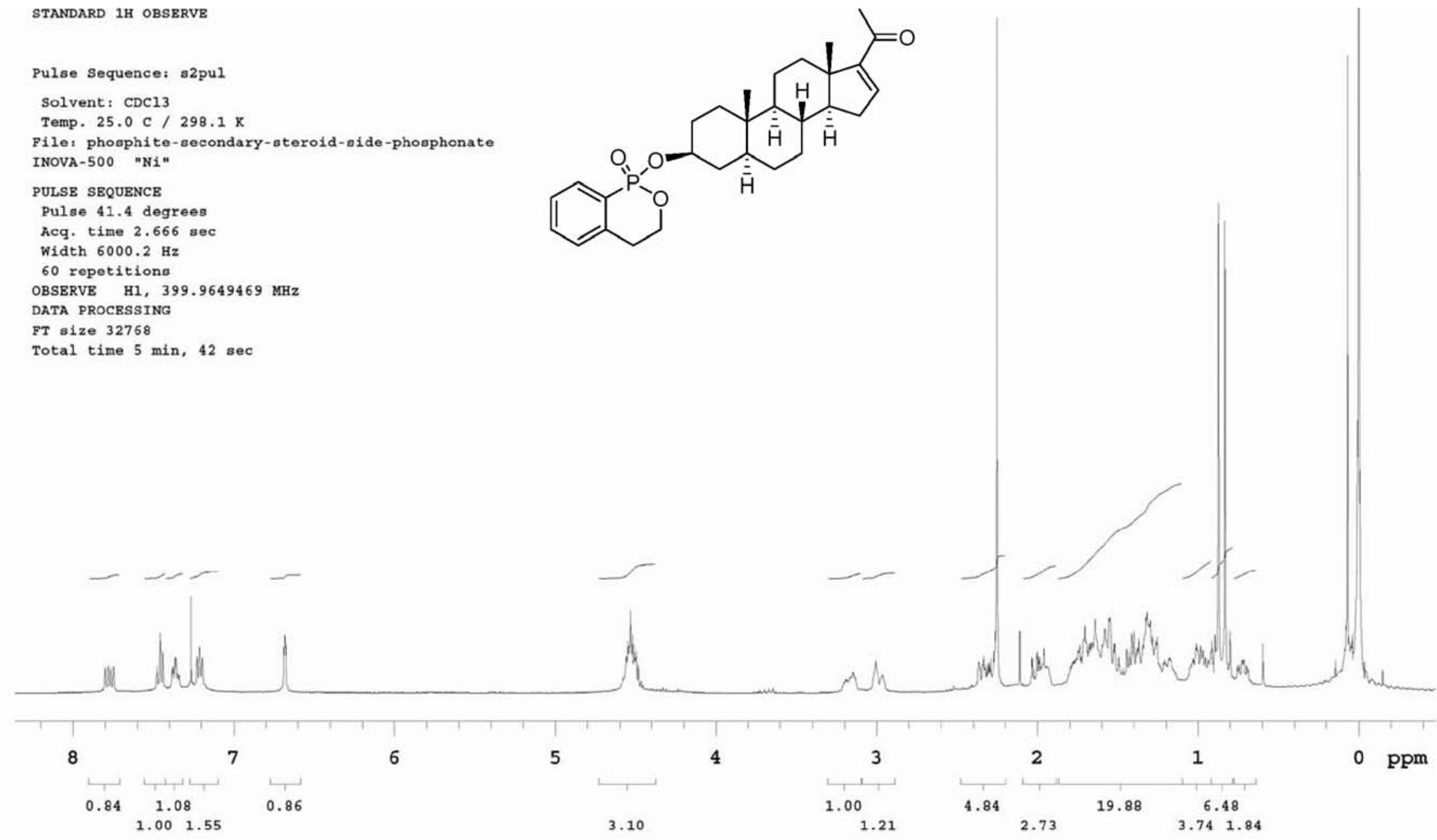
PHOSPHORUS OBSERVE

STANDARD PARAMETER

Pulse Sequence: s2pul

Solvent: $\mathrm{CDC} 13$

Temp. $25.0 \mathrm{C} / 298.1 \mathrm{x}$

ile: phosphite-phosphonate-side-product- $p^{3}$

INOVA-500 "Ni"

PULSE SEQUENCE

Pulse 44.2 degrees

Acq. time 0,800 sec

width 50000.0 .

Width 50000.0 Hz

232 repetitions

BSERV

$44 \mathrm{MHz}$

Power $44 \mathrm{~dB}$

continuously on

WALTZ-16 modulated

DATA PROCESSING

Line broadening $1.0 \mathrm{~Hz}$

T size 131072

Total time $2 \mathrm{hr}, 52 \mathrm{~min}, 33 \mathrm{sec}$

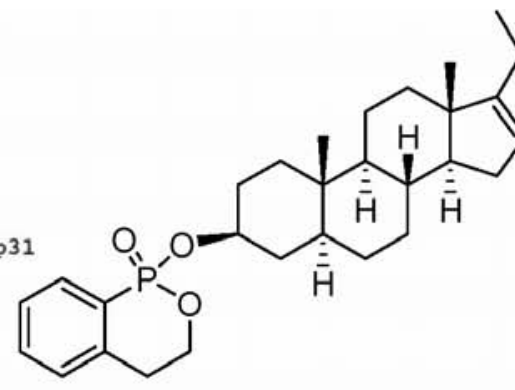

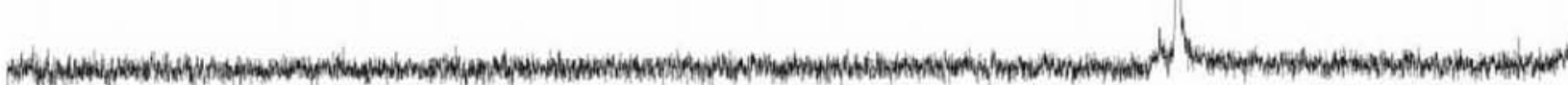

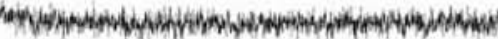


STANDARD 1H OBSERVE

Pulse Sequence: s2pul

Solvent: $\mathrm{CDC13}$

Temp. $25.0 \mathrm{C} / 298.1 \mathrm{x}$

File: phosphite-phosphonate

INOVA-500 "Ni"

PULSE SBQUENCE

Pulse 41.4 degrees

Acq. time $2.666 \mathrm{sec}$

width $6000.2 \mathrm{~Hz}$

32 repetitions

OBSERVE H1, $399.9649454 \mathrm{MHz}$

DATA PROCESSING

T size 32768

rotal time $5 \mathrm{~min}, 42 \mathrm{sec}$

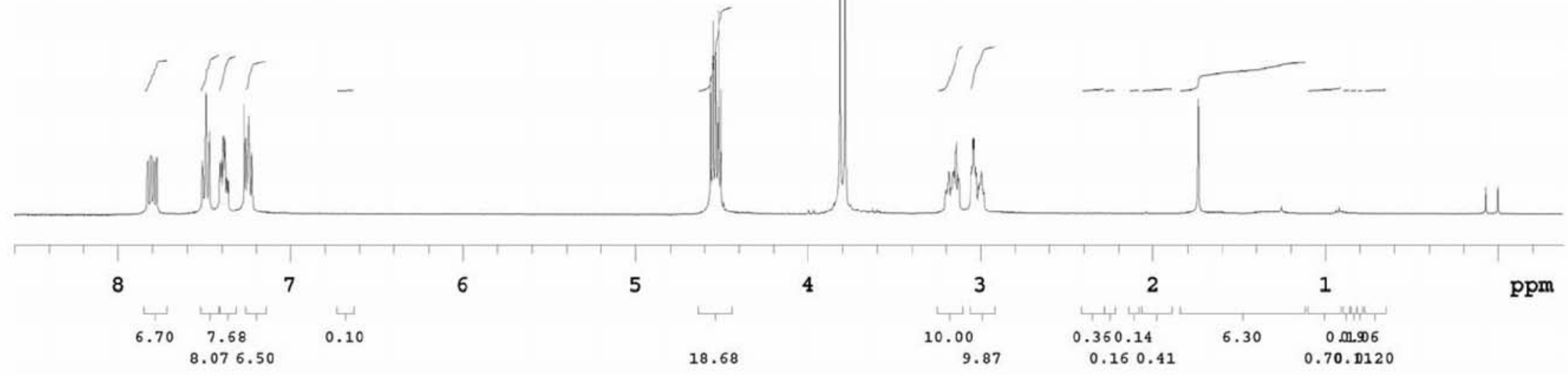


PHOSPHORUS OBSERVE

STANDARD PARAMETERS

PHOSPHATE REGION

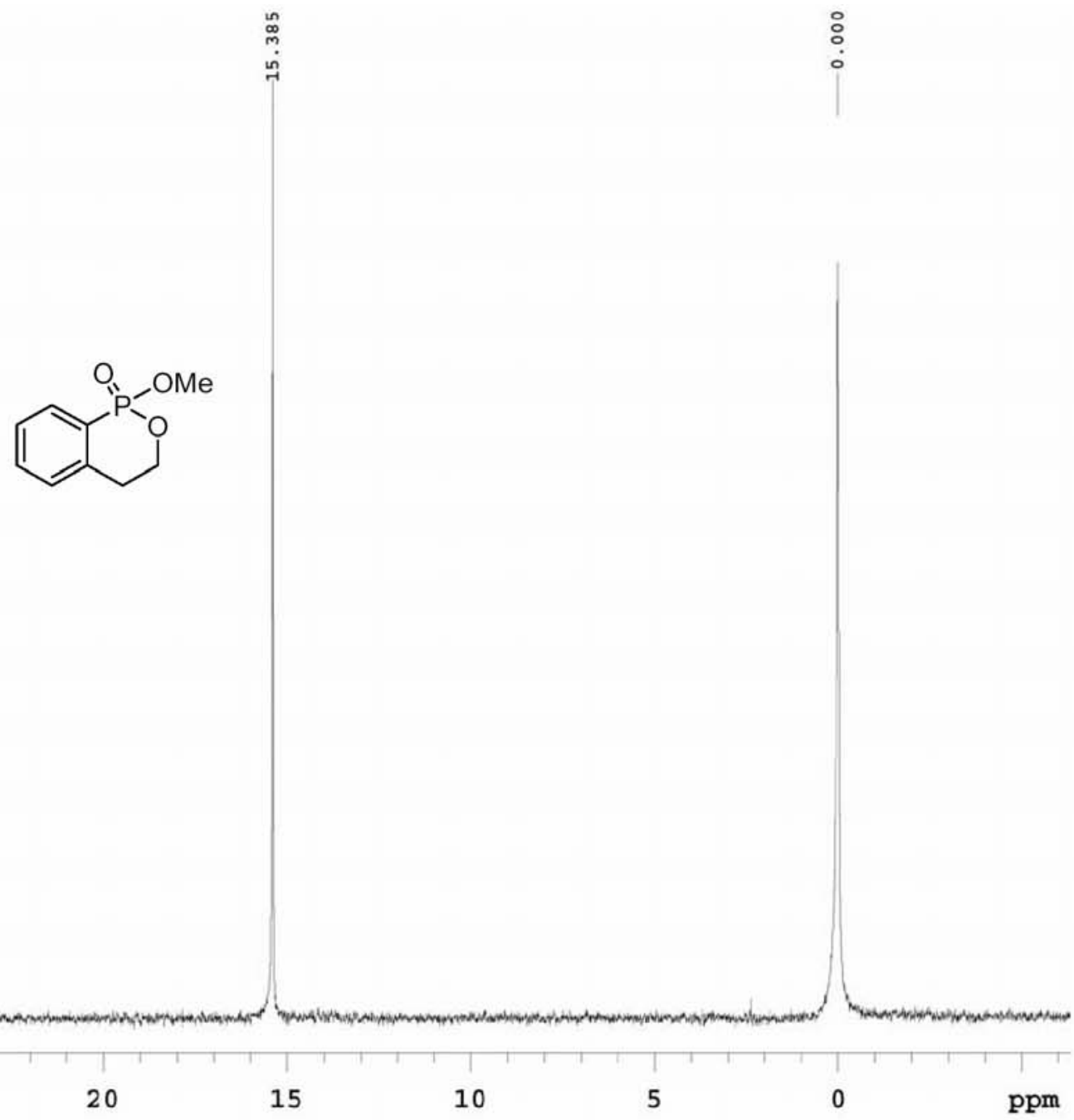


STANDARD $1 \mathrm{H}$ OBSBRVE

Pulse Sequence: s2pul

Solvent: $\mathrm{CDC} 13$

Ambient temperature

File: p-steroid-h

INOVA-500 "Ni"

PULSE SEQUENCE

Pulse 41.4 degrees

Acq. time $2.666 \mathrm{se}$

Width $3759.8 \mathrm{~Hz}$

32 repetitions

OBSERVE H1, $399.9649459 \mathrm{MHz}$

DATA PROCESSING

FT size 32768

Total time $1 \mathrm{~min}, 25 \mathrm{sec}$

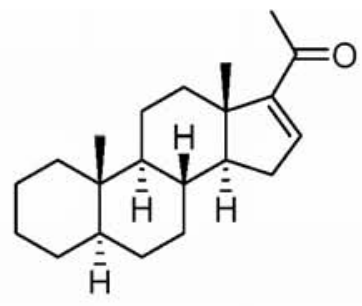

$\overline{\mathrm{H}}$

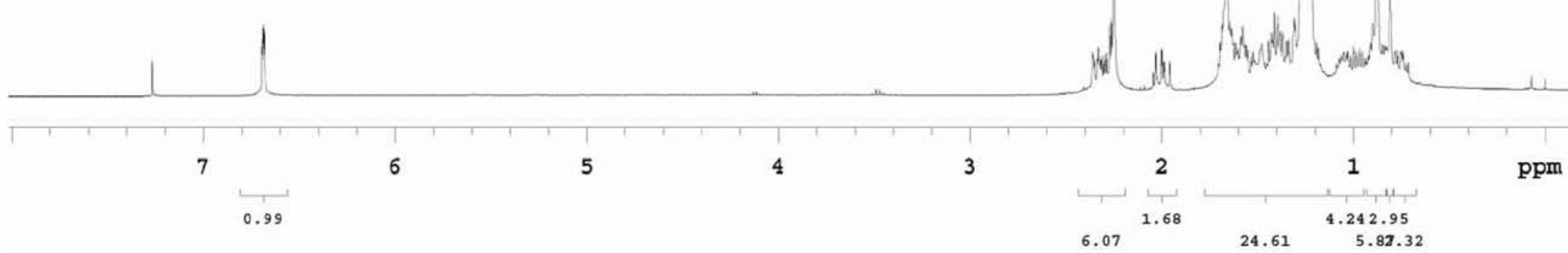


STANOARD CARBON PARAMCTERS Pulse Sequence: s2pul Solyent: CoC13

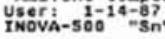

PuLsE SEGue HCE

Acg. ${ }^{2} 11001.300 \mathrm{sec}$

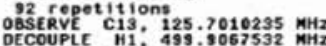
cont

\section{TT.}

Total time 6 hr, $41=1 n, 52 \mathrm{sec}$

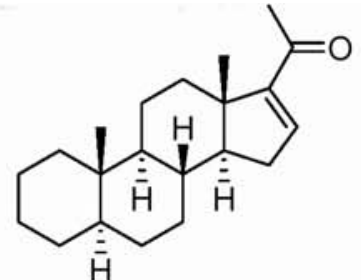

$\overline{\mathrm{H}}$

$9582.470-77.283$

5940.787
55.216

5823.030
4833.912
38.456

$\begin{array}{ll}4378.023 & 34.829\end{array}$

$4247.123 \quad 33.787$

$\begin{array}{ll}\begin{array}{l}4027.421 \\ 3652.553\end{array} & 32.040 \\ 30.057 & -052\end{array}$

$\begin{array}{lll}3636.931 & 28.934 \\ 3449.159 & 27.959\end{array}$

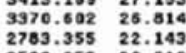

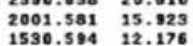

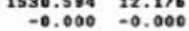

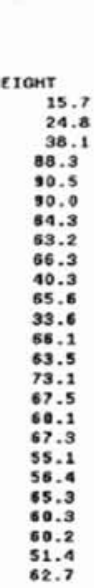

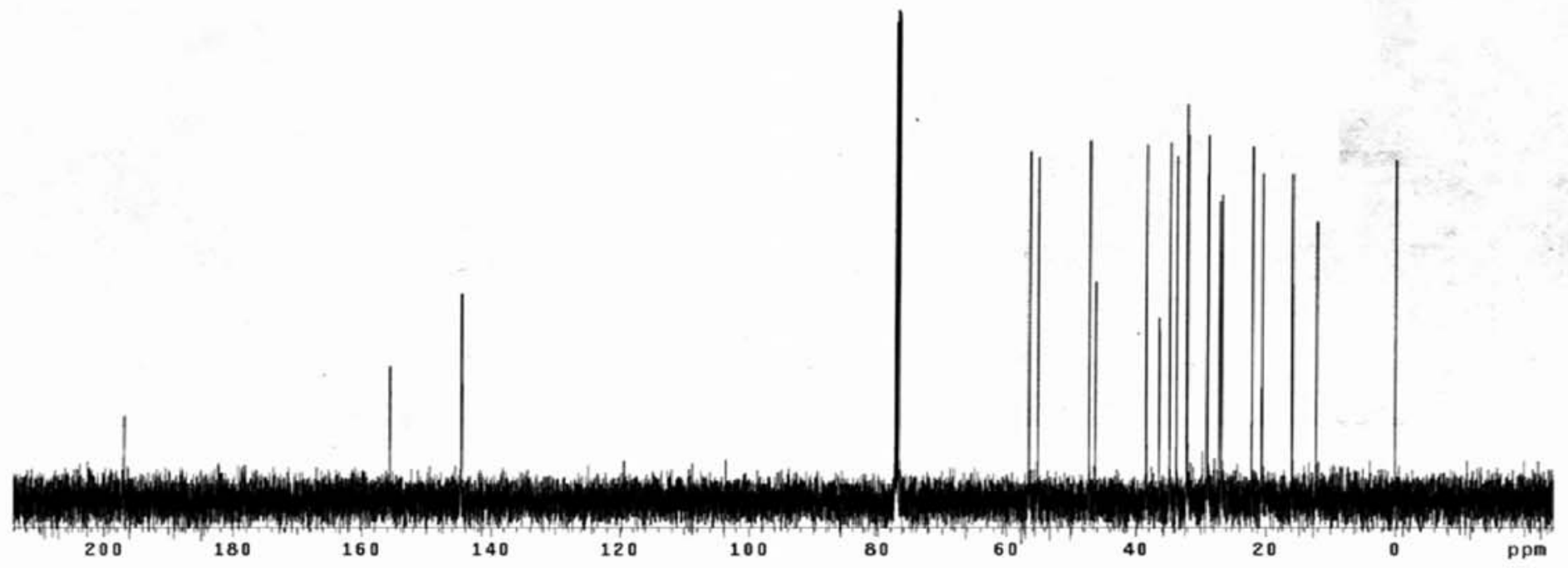




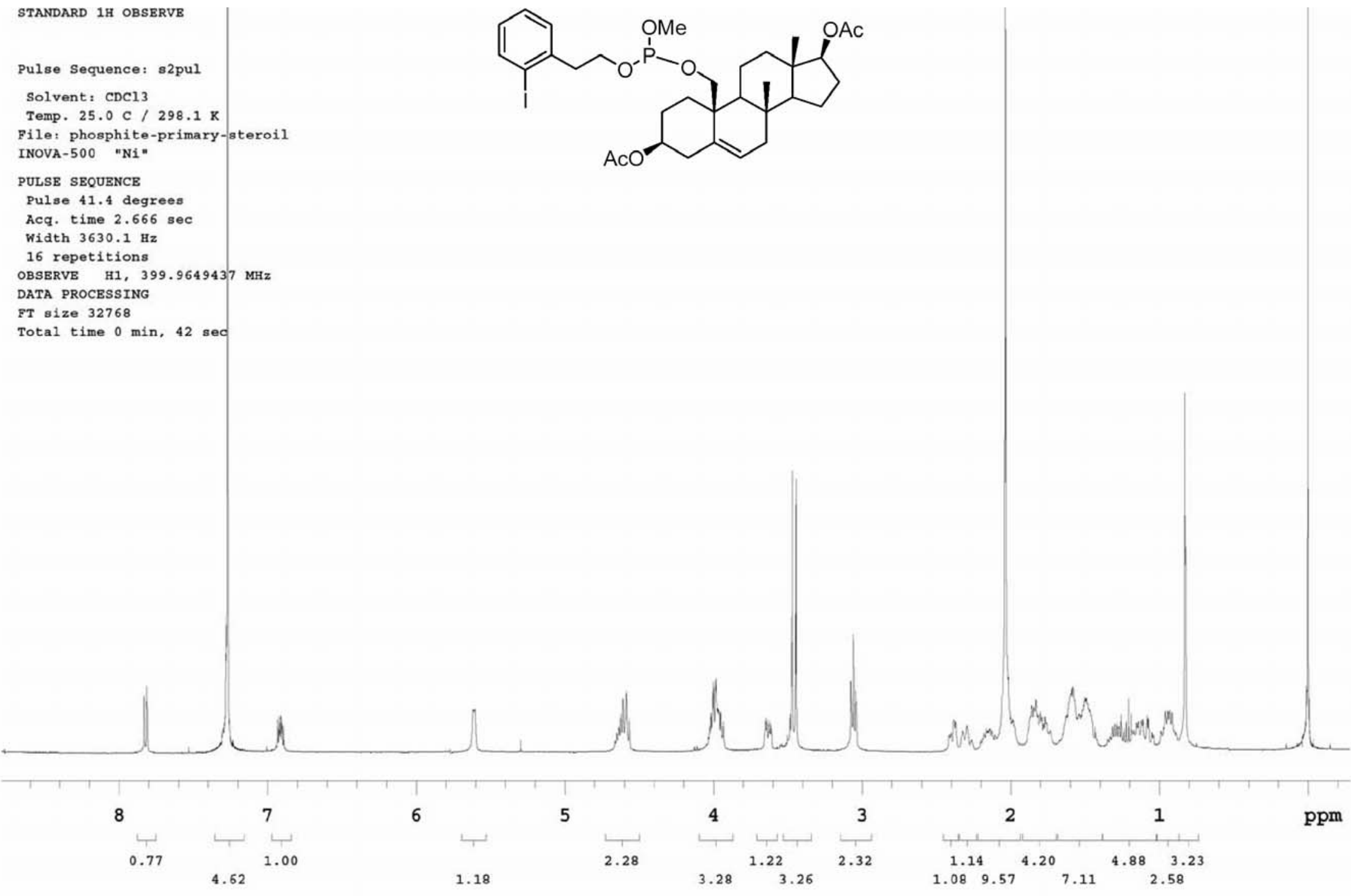


13C OBSERVE

Pulse Sequence: s2pul

Solvent: $\mathrm{CDC1} 3$

Ambient temperature

imary-steroid-c13

INOVA-500 "Ni"

PULSE SEQUENCE

Relax. delay $0.900 \mathrm{sec}$

Pulse 45.0 degrees

Acq. time $1.199 \mathrm{sec}$

width $25000.0 \mathrm{~Hz}$

608 repetitions

OBSERVE C13, 100.5712646 MHz

DECOUPLE H1, $399.9669644 \mathrm{MHz}$

Power $44 \mathrm{~dB}$

continuously on

WALTZ-16 modulated

DATA PROCESSING

Line broadening $1.0 \mathrm{Hig}$

FT size 65536

Total time $70 \mathrm{hr}, 16$ ming $25 \mathrm{sec}$

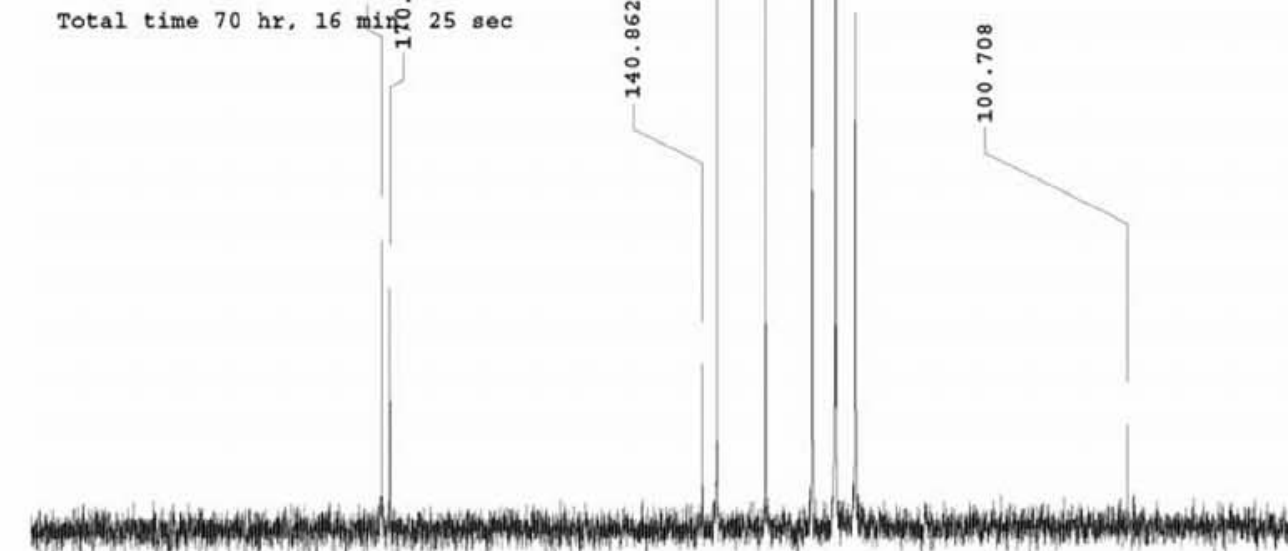

200

180

160

140

120

100

60

40

ppm 


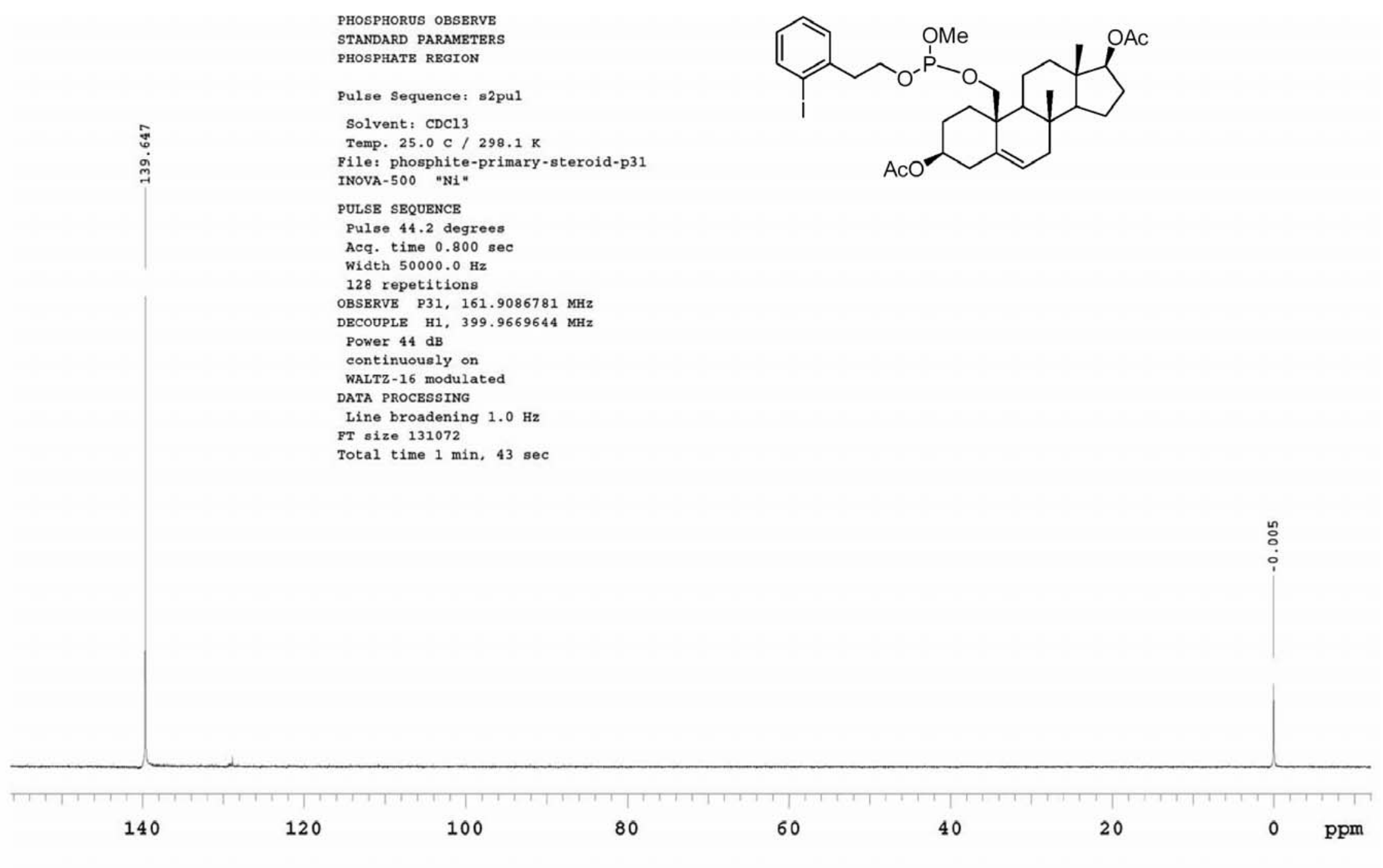


STANDARD $1 \mathrm{H}$ OBSERVE

Pulse Sequence: s2pul

Solvent: $\mathrm{CDC} 13$

Temp. $25.0 \mathrm{C} / 298.1 \mathrm{~K}$

File: phosphite-primary-steroid-reduction-pure

NOVA-500 "Ni.

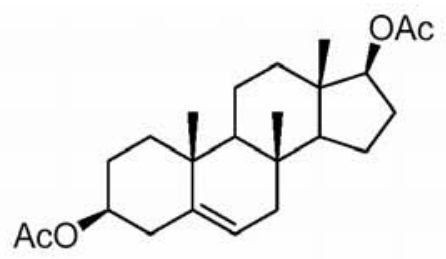

ULSE SEQUENCE

Pulse 41.4 degrees

Acq. time $2.666 \mathrm{sec}$

width $3630.1 \mathrm{~Hz}$

32 repetitions

OBSERVE H1, $399.9649468 \mathrm{MHz}$

DATA PROCESSING

FT size 32768

Total time $1 \mathrm{~min}, 25 \mathrm{sec}$

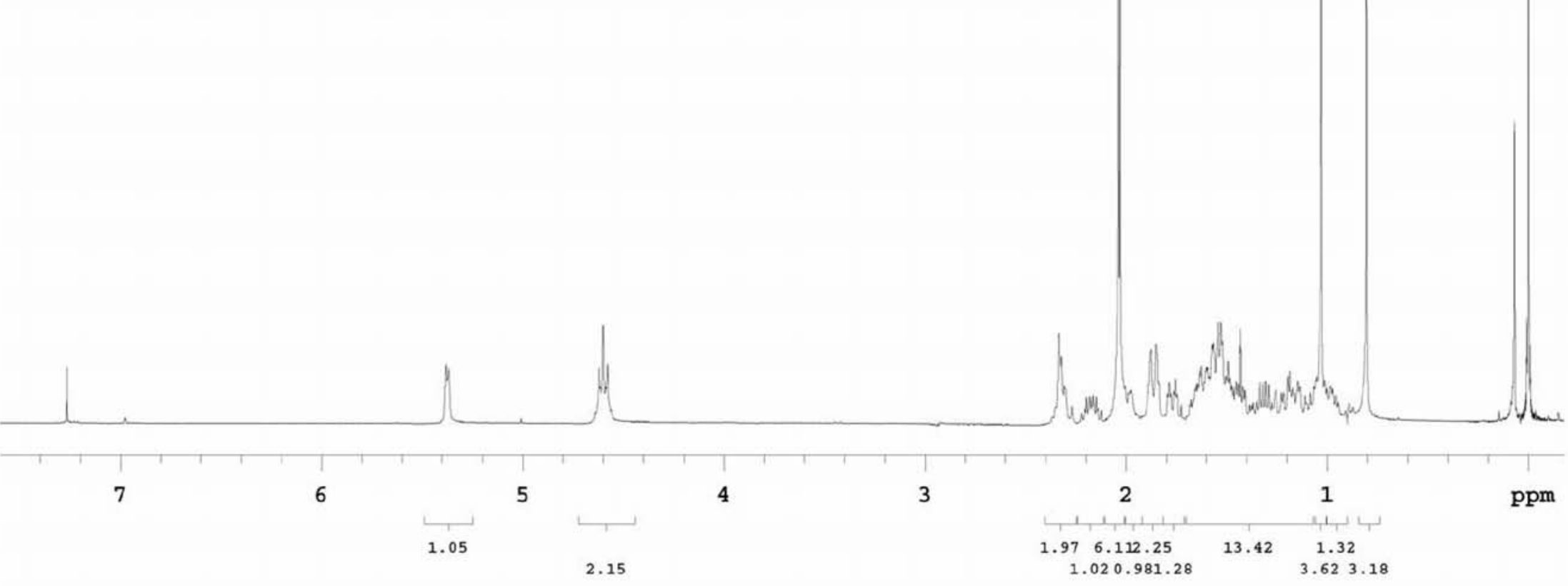


13C OBSERVB

Pulse Sequence: s2pul

Solvent: $\mathrm{CDC} 13$

Ambient temperature

ile: phosphite-primary-steroid-reduction-pure-c13

INOVA-500 "Ni"

PULSE SEQUENCE

Relax. delay $0.900 \mathrm{sec}$

Pulse 45.0 degrees

Acq. time $1.199 \mathrm{sec}$

Width $25000.0 \mathrm{~Hz}$

544 repetitions

OBSERVE C13, 100.5712631 MHz

BCOUPLB H1, $399.9669644 \mathrm{MHz}$

Power $44 \mathrm{~dB}$

continuously on

WALTZ - 16 modulated

DATA PROCESSING

Line broadening $1.0 \mathrm{~Hz}$

T size 65536 Total time $70 \mathrm{hr}, 16 \mathrm{~min}, 25 \mathrm{~h}$

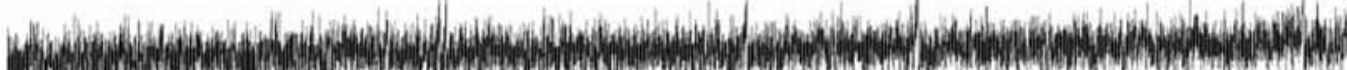

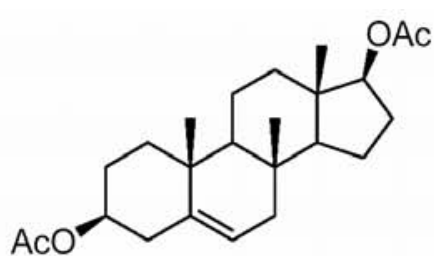

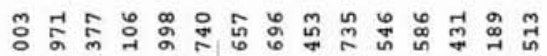

ก่

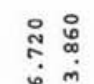

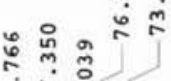

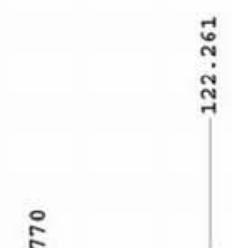

di :

$\stackrel{\text { m }}{\sim}$
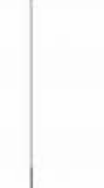

mint

200

180

160

140

120

100

80

60

40

20

ppm 
STANDARD $1 \mathrm{H}$ OBSBRVE

Pulse Sequence: s2pul

Solvent: $\mathrm{CDC} 13$

Ambient temperature

File: phosphite-cis-tbutylcyclohexanol

fle: phosphite-ci

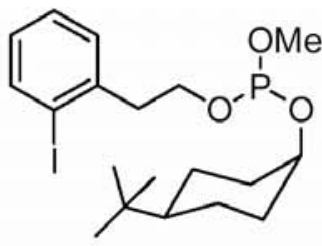

PULSE SEQUENCE

Pulse 42.1 degrees

Acq. time $3.333 \mathrm{sec}$

Width $4500,5 \mathrm{~Hz}$

32 repetitions

32. 300.0732307

PROCESSING

Line broadening $0.2 \mathrm{~Hz}$

FT size 32768

Total time $1 \mathrm{~min}, 46 \mathrm{sec}$

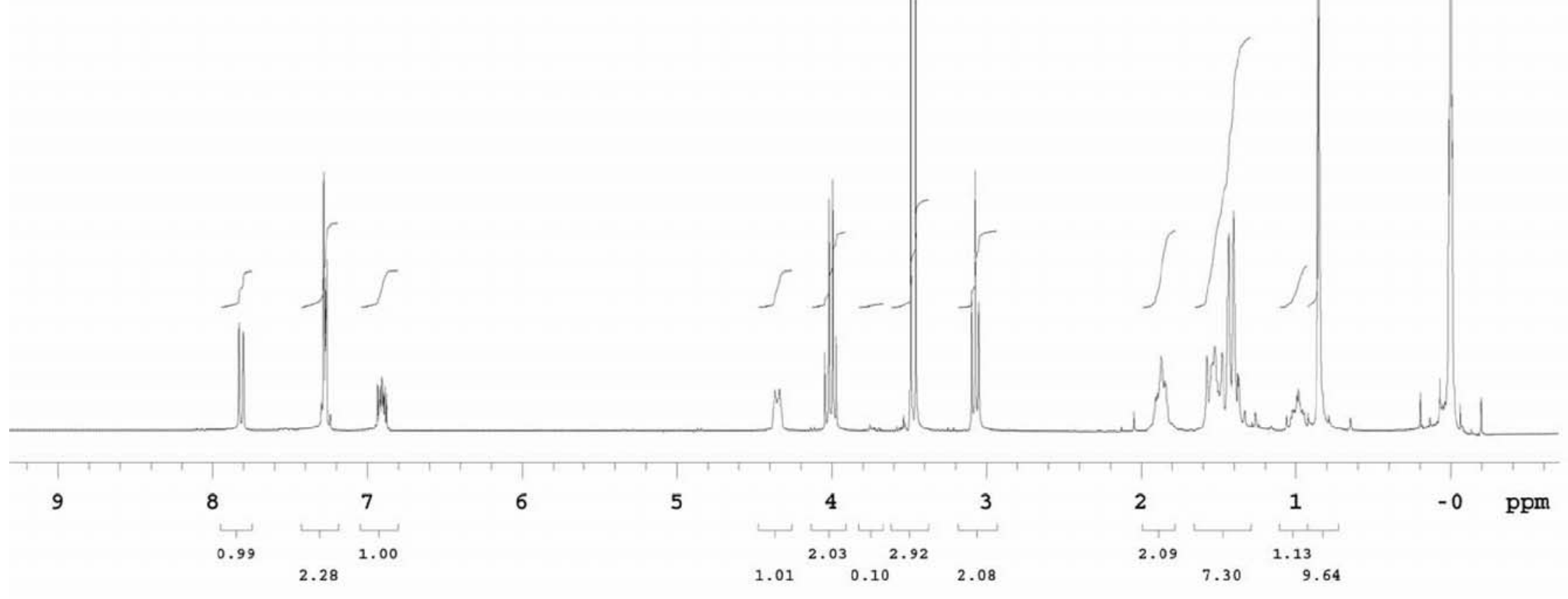


STANDARD CARBON PARAMETERS

Pulse Sequence: s2pul

Solvent: $\mathrm{CDC13}$

Ambient temperature

User: 1-14-87

File: phosphite-cis-tbucyclohexanol-c

INOVA- 500 "Ni"

PULSE SEQUENCE

Relax. delay 0,700 sec

Pulse 27.2 degrees

Acq. time $1.300 \mathrm{sec}$

Width $29996,3 \mathrm{~Hz}$

256 repetitions

OBSERVE C13,125.7010245 MH

ECOUPLE H1, 499.9067532 MHz

Power $32 \mathrm{~dB}$

continuously on

WALTZ - 16 modulated

DATA PROCESSING

Line broadening $0.5 \mathrm{~Hz}$

size 131072

Total time $6 \mathrm{hr}, 41 \mathrm{~min}, 52 \mathrm{sec}$<smiles>COP1OC2CCC(C(C)(C)C)CCC(C2)O1</smiles>

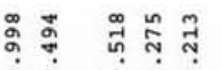

이 임

Tर 1 T

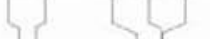

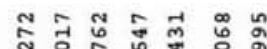

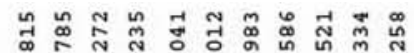

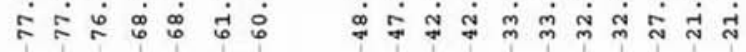

$\vdots$
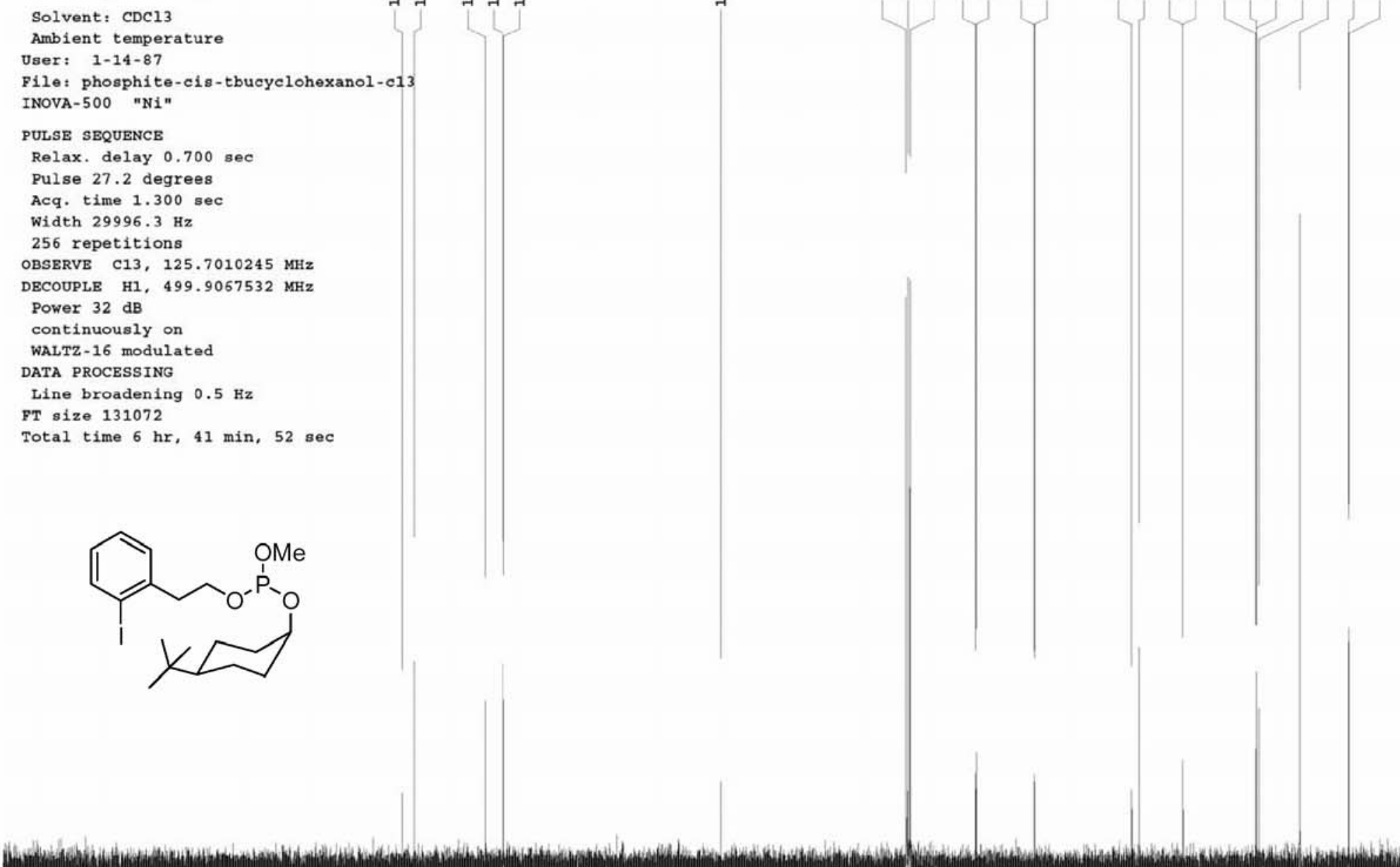

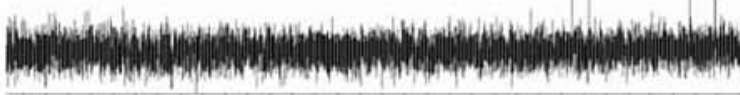

180

160

140

120

100

80

60

40

20

ppm 
PHOSPHORUS OBSERVE

STANDARD PARAMETERS

PHOSPHATE REGISN

Pulse Sequence: a2pul

Solvent: $\mathrm{CDC1} 3$

Temp. $25.0 \mathrm{C} / 298.1 \mathrm{~K}$

pile: phoaphite-cis-tbutycyclohexanol-p31

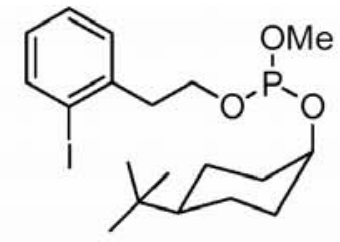

INOVA- 500 "Ni

PULSE SEQUENCE

Pulse 44.2 degrees

Acq. time $0.800 \mathrm{sec}$

Acq. time 0.800 sec

84 repetition

P31, 161.9086874 MHz

ECOUPLE H1, $399.9669644 \mathrm{MHz}$

Power $44 \mathrm{~dB}$

continuously on

WALTZ-16 modulated

DATA PROCESSING

Line broadening $1.0 \mathrm{~Hz}$

FT size 131072

Total time $1 \mathrm{~min}, 43 \mathrm{sec}$

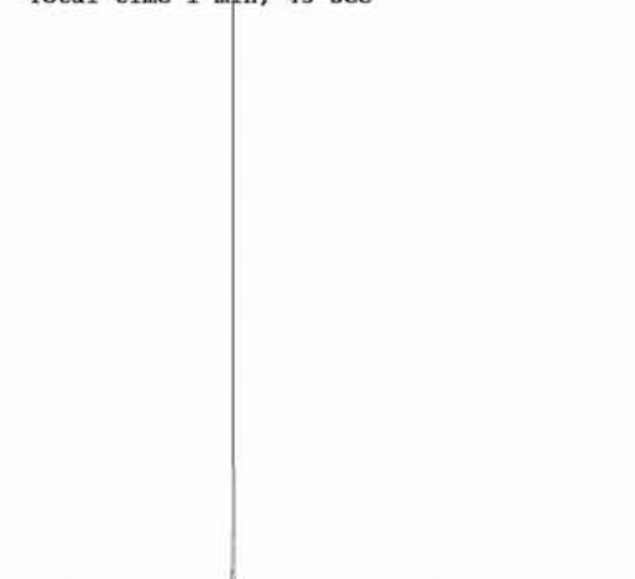

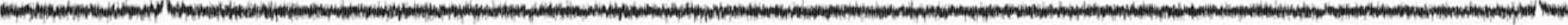

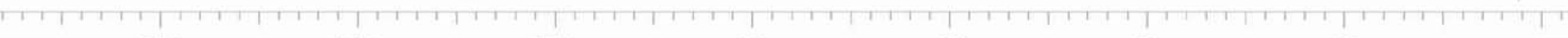


STANDARD $1 \mathrm{H}$ OBSERVE

Pulse Sequence: s2pul

Solvent: $\mathrm{CDCl} 3$

Temp. $25.0 \mathrm{C} / 298.1 \mathrm{~K}$

File: phosphite-3-octanol

INOVA-500 "Ni"

PULSE SEQUENCE

Pulse 41.4 degrees

Acq. time 2.666 sec

width $3630.1 \mathrm{~Hz}$

16 repetitions

DATA PROCESSING

T size 32768

Total time $0 \mathrm{~min}, 42 \mathrm{sec}$
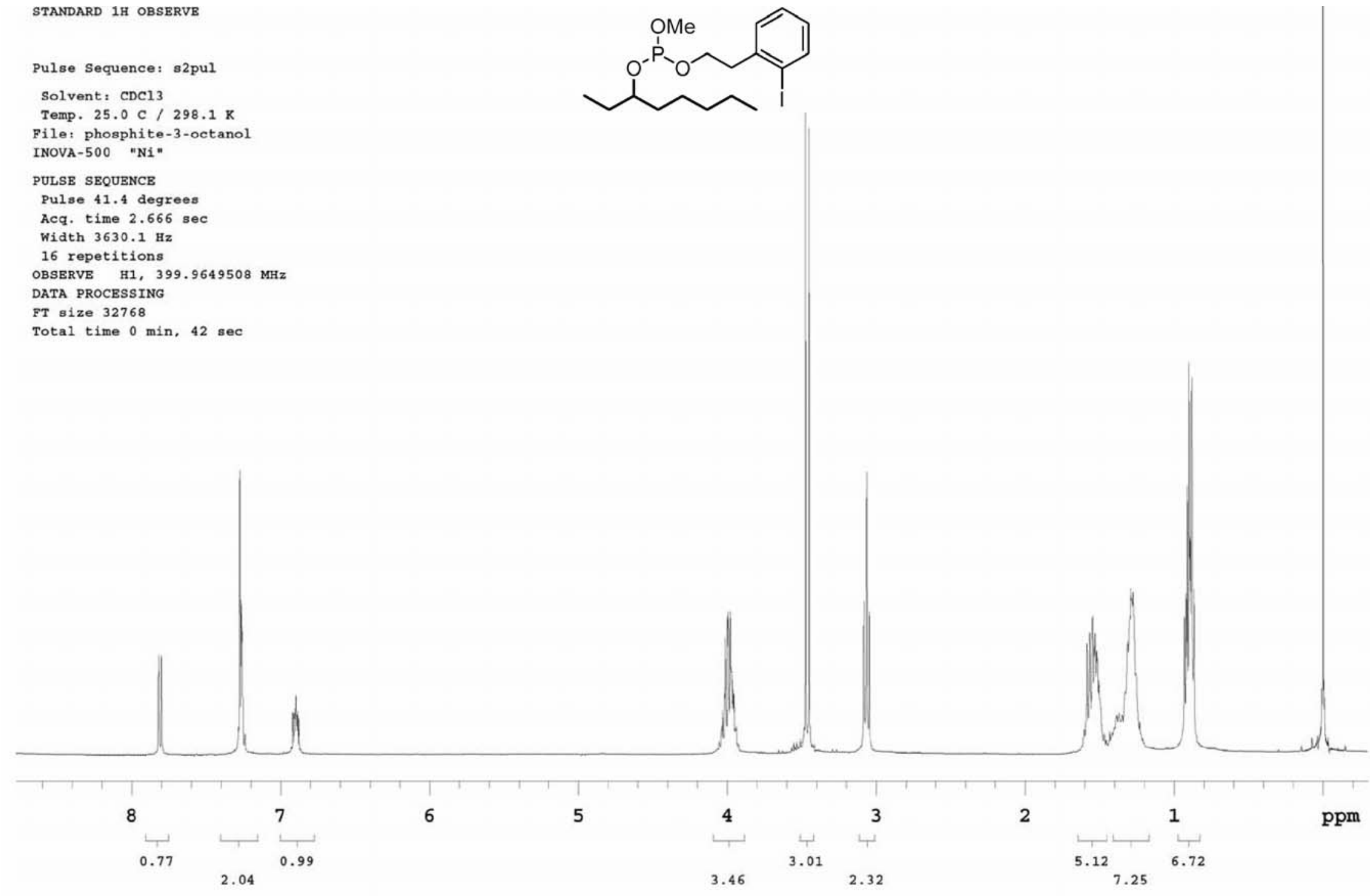
13C OBSERVE

Pulse Sequence: s2pul

Solvent: $\mathrm{CDCl} 3$

Temp. $25.0 \mathrm{C} / 298.1 \mathrm{~K}$

File: phosphite-3-octanol-p31

INOVA-500 "Ni"

PULSE SEQUENCE

Relax. delay $0.900 \mathrm{sec}$

Pulse 45.0 degrees

Acq. time $1.199 \mathrm{sec}$

width $25000.0 \mathrm{~Hz}$

288 repetitions

OBSERVE C13,100.5712654 MHz

DECOUPLE H1, 399.9669644 MHZ

Power $44 \mathrm{~dB}$

continuously on

WALTZ-16 modulate

DATA PROCESSING

Line broadening $1.0 \mathrm{~Hz}$

T size 65536

Total time $70 \mathrm{hr}, 16 \mathrm{~min}, 25 \mathrm{sec}$
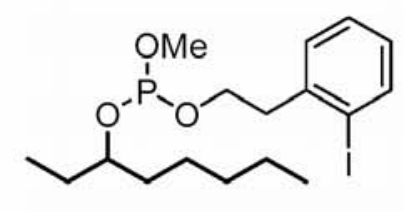<smiles>[Tl]</smiles>

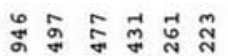

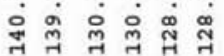

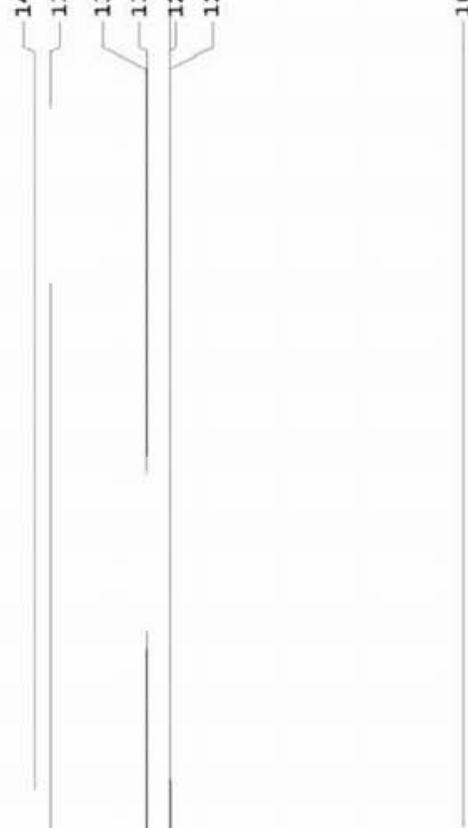

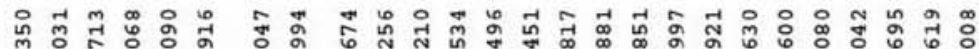

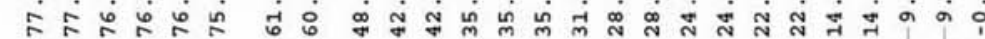

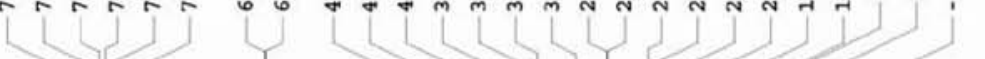

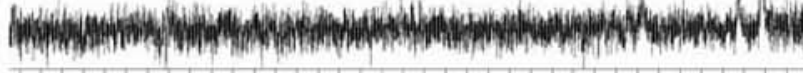

180

160

140

120

100

80

60

40

20

ppm 
PHOSPHORUS OBSERVE

STANDARD PARAMETERS

PHOSPHATE REGION

$\begin{array}{lllr}\text { NUEX } & \text { FKEQUENCY } & \text { YYM } & \text { HEIGHT } \\ 1 & 22785.534 & 140.731 & 94.3\end{array}$

Pulse Sequence: s2pul

Solvent: $\mathrm{CDC1} 3$

Temp. $25.0 \mathrm{C} / 298.1 \mathrm{~K}$

File: phosphite-3-octanol-c13

INOVA - 500 "Ni"

PULSE SEQUENCE

Pulse 44.2 degrees

Acg. time 0,800 sec

Acq. time $0.800 \mathrm{sec}$

We $5000.0 \mathrm{~Hz}$

OBSERVE P31, 161.9086766 MHz

\begin{tabular}{lr|ll} 
OBSERVE P31, & $161.9086766 \mathrm{MHz}$ \\
DECOUPLE H1, & $399.9669644 \mathrm{MHz}$
\end{tabular}

Power $44 \mathrm{~dB}$

continuously on

WALTZ-16 modulate

DATA PROCESSING

Line broadening $1.0 \mathrm{~Hz}$

FT size 131072

Total time 1 min, $43 \mathrm{sec}$

$\begin{array}{llll}1 & 22780.194 & 140.698 & 83.9\end{array}$
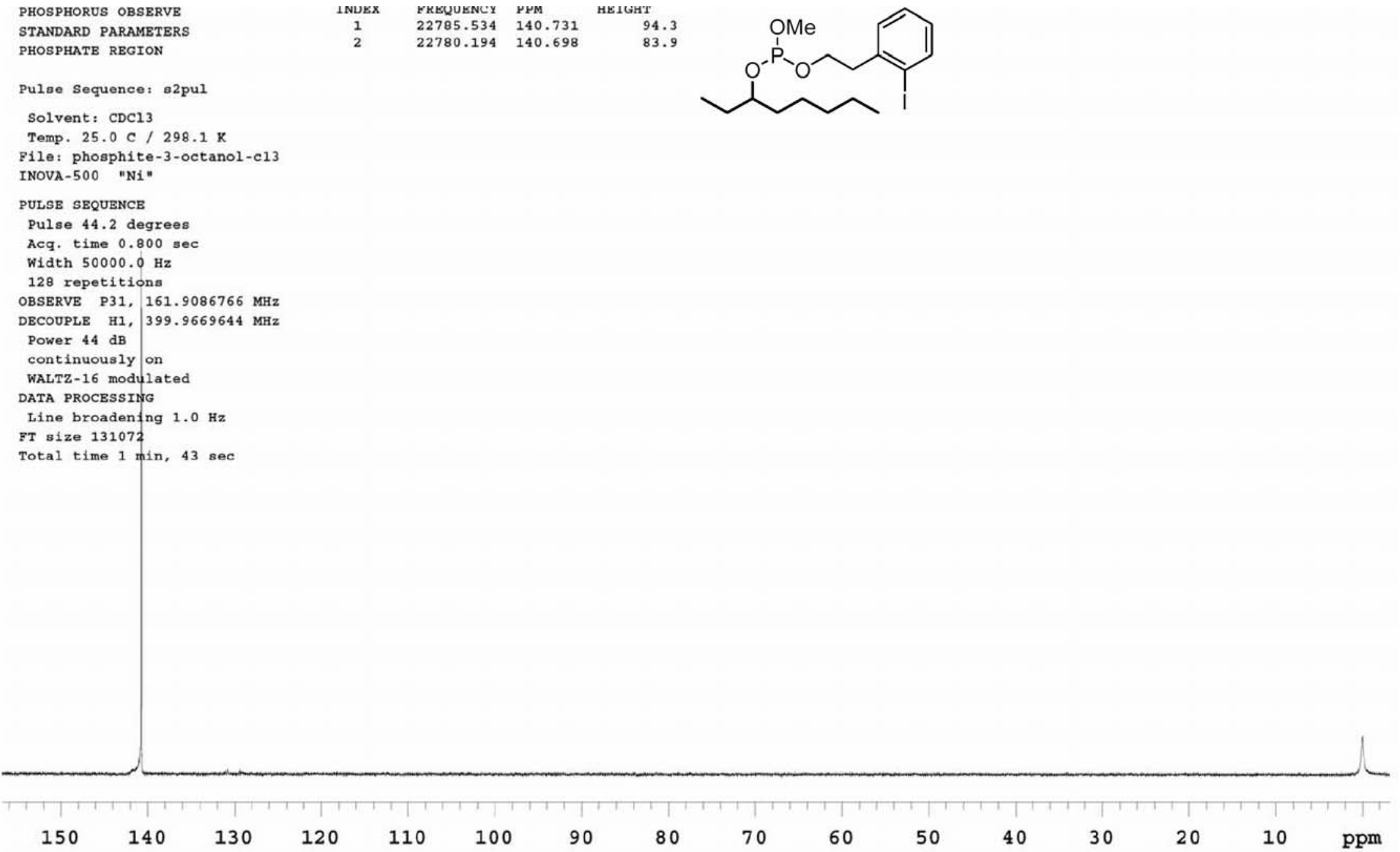
STANDARD 1H OBSERVE

Pulse Sequence: s2pul

Solvent: $\mathrm{CDCl} 3$

Temp. $25.0 \mathrm{C} / 298.1 \mathrm{~K}$

ile: phosphite-secondary-chain-alcohol-alpha-methy

INOVA-500 "Ni"

PULSE SEQUENCE

Pulse 41.4 degrees

Acq. time $2.666 \mathrm{sec}$

Width $3630.1 \mathrm{~Hz}$

32 repetitions

$61 \mathrm{MHz}$

DATA PROCESSING

FT size 32768

Total time $1 \mathrm{~min}, 25 \mathrm{sec}$
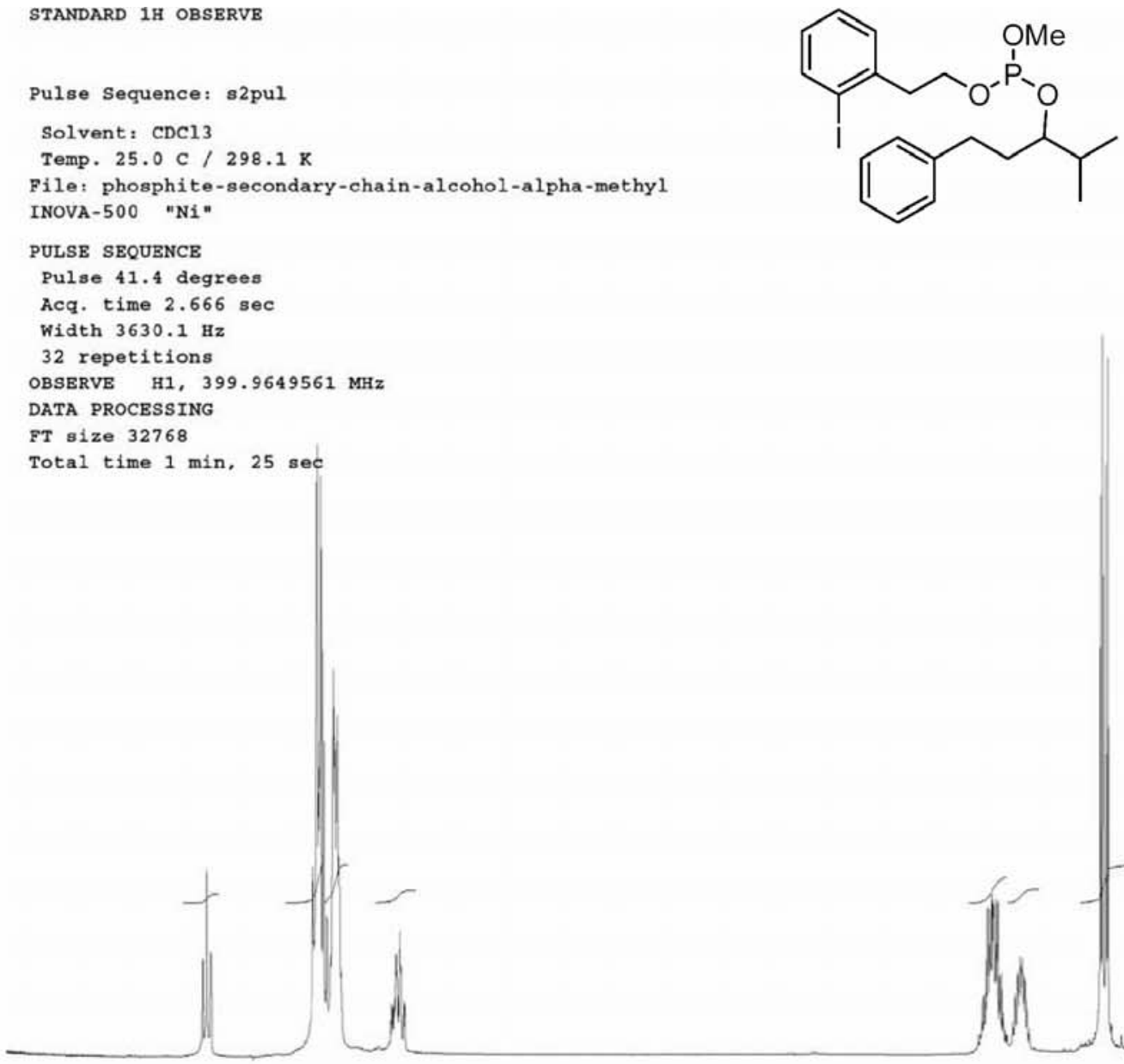

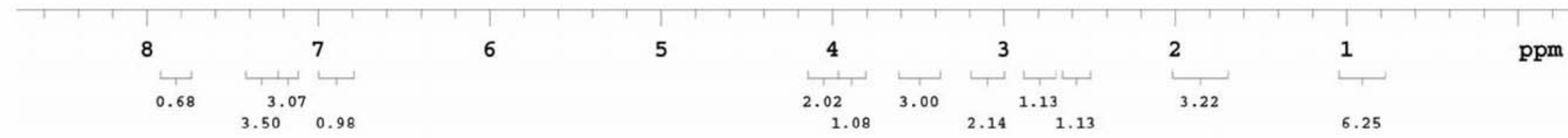


PHOSPHORUS OBSERVE

STANDARD PARAMETERS

PHOSPHATE REGION

Pulse Sequence: s2pul

Solvent: $\mathrm{CDCl}_{3}$

Temp. $25.0 \mathrm{C} / 298.1 \mathrm{~K}$

ile: phosphite-secondary-alcohol-chain-alpha-methy1-p3

PULSE SEQUENCE

Pulse 44.2 degrees

Acq. time $0.800 \mathrm{sec}$

Width $60015.0 \mathrm{~Hz}$

104 repetitions

OBSERVE P31, $161.9086854 \mathrm{MH}$

DECOUPLE H1, $399.9669644 \mathrm{MHz}$

Power $44 \mathrm{~dB}$

continuously on

WALTZ-16 modulate

DATA PROCESSING

Line broadening $1.0 \mathrm{~Hz}$

FT size 131072

Total time $2 \mathrm{hr}, 52 \mathrm{~min}, 33 \mathrm{sec}$

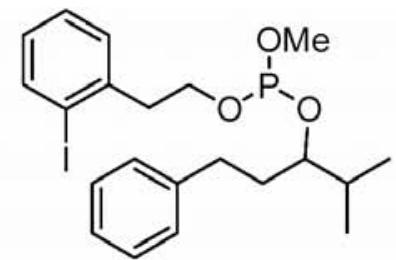

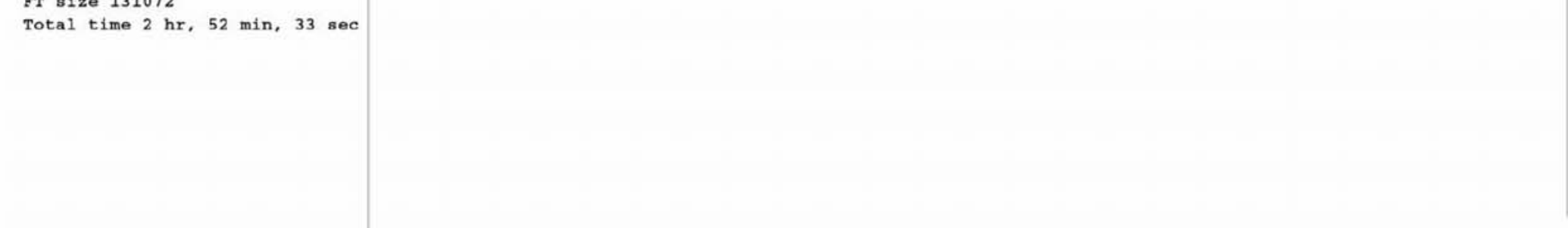


STANDARD CARBON PARAMETERS

Pulse Sequence: s2pul

Solvent: $\mathrm{CDC} 13$

Ambient temperature

File: phosphite-sugar

INOVA-500 "Ni"

\section{PULSE SEQUENCE}

Relax. delay $1.000 \mathrm{sec}$

Pulse 44.2 degrees

Acq. $t$ ime $2.500 \mathrm{sec}$

Width $4671.8 \mathrm{~Hz}$

8 repetitions

41, 499.9042537 $\mathrm{MHz}$

T)
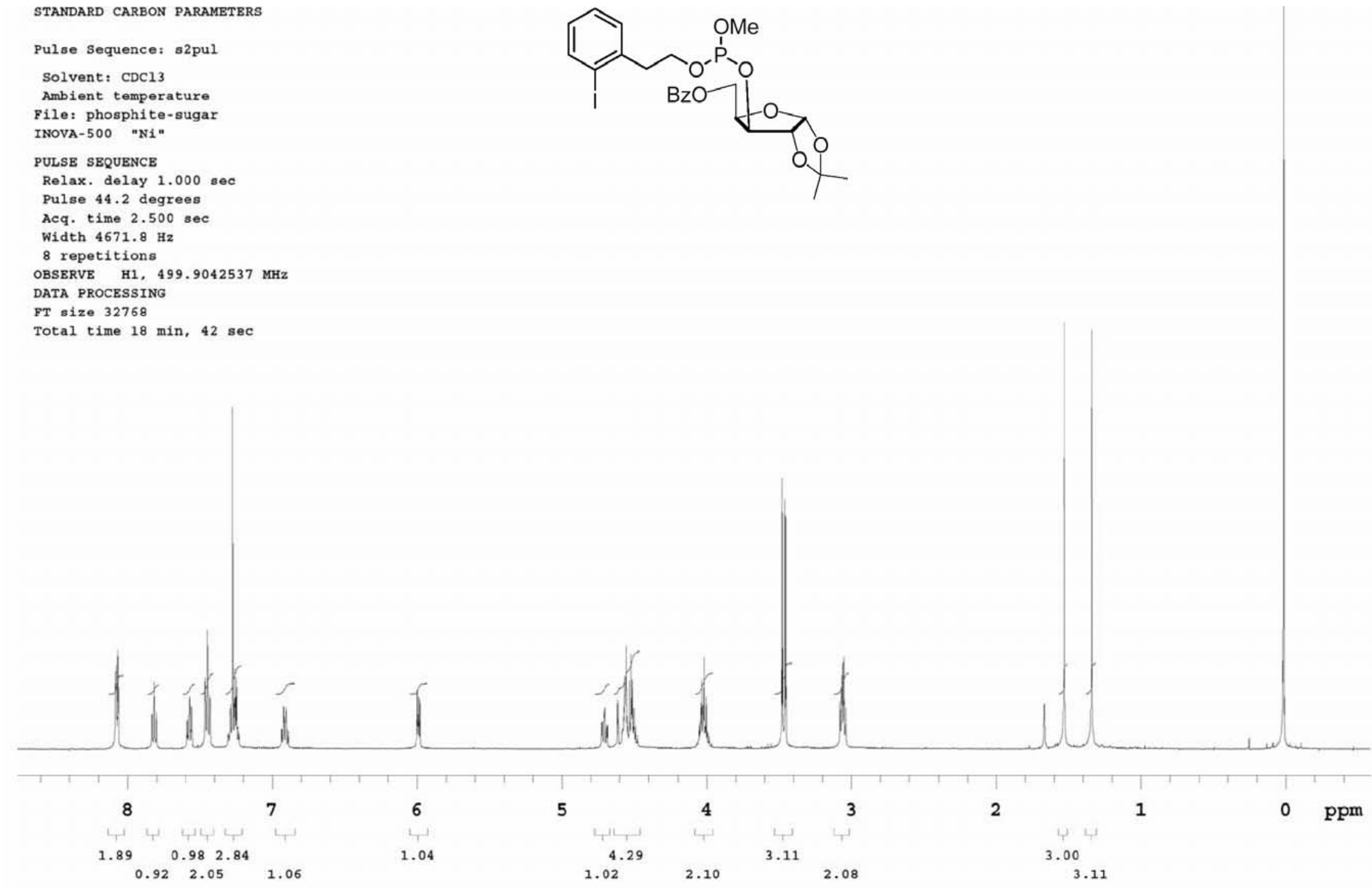
PHOSPHORUS OBSBRVB

STANDARD PARAMETERS

PHOSPHATE REGION

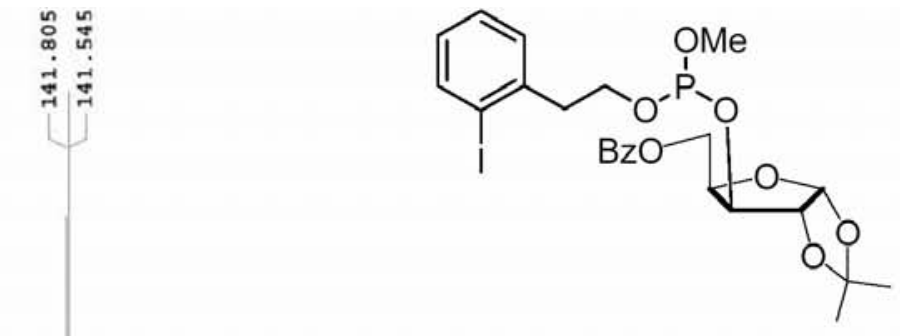

Pulse Sequence: s2pul

Solvent: $\mathrm{CDC} 13$

Ambient temperature

File: phosphite-sugar-p31

INOVA-500 "Ni"

PULSE SEQUENCB

Pulse 45.0 degrees

Acq. time $0.800 \mathrm{se}$

Width $60015.0 \mathrm{~Hz}$

128 repetitions

OBSERVE P31, $161.9086047 \mathrm{MHz}$

DECOUPLE H1, $399.9669644 \mathrm{MHz}$

Power $44 \mathrm{~dB}$

continuously on

WALIZ-16 modulated

DATA PROCESSING

Line broadening $1.0 \mathrm{~Hz}$

FT size 131072

Total time $1 \mathrm{~min}, 43 \mathrm{sec}$

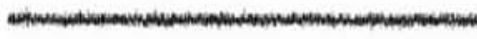

180

160

140

120

100

80

60

40

20

ppm 
STANDARD 1H OBSERVE

Pulse Sequence: s2pul

Solvent: CDC13

Ambient temperature

File: p-sugar-h

INOVA-500 "Ni"

PULSE SEQUENCE

Pulse 41.4 degrees

Acq. time $2.666 \mathrm{sec}$

width $3759.8 \mathrm{~Hz}$

16 repetitions

OBSERVE H1, $399.9649473 \mathrm{MH}$

DATA PROCESSING

FT size 32768

Total time $1 \mathrm{~min}, 25 \mathrm{sec}$
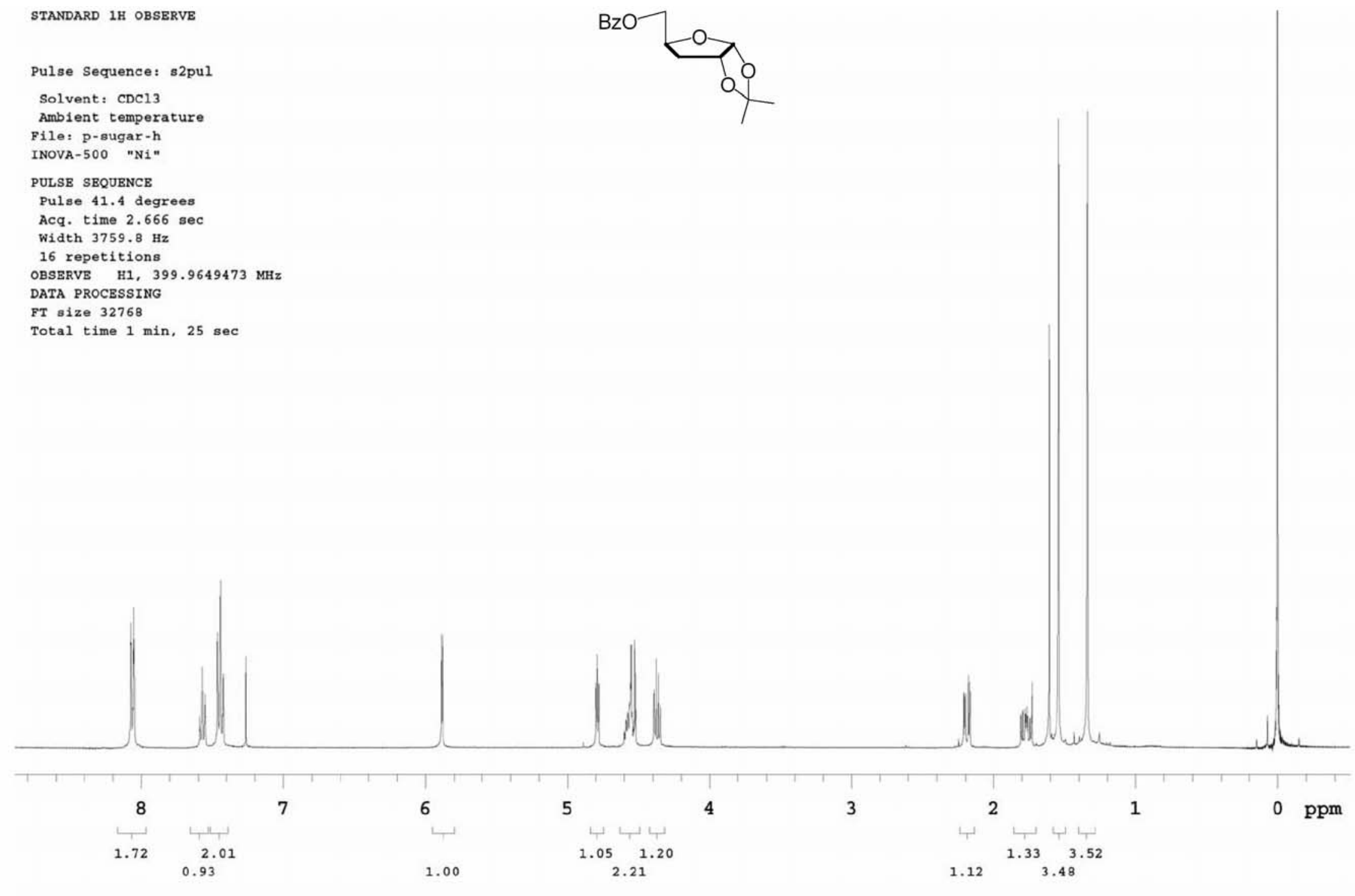


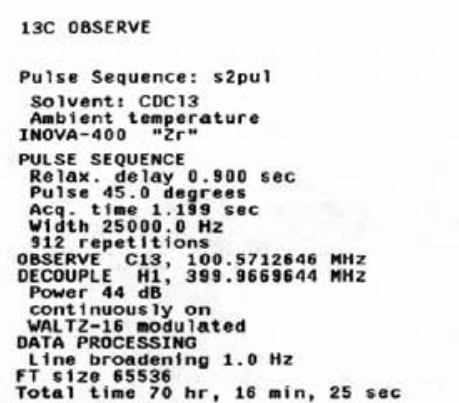

$$
\begin{gathered}
\text { INDEX } \\
1 \\
2 \\
3 \\
4 \\
5 \\
5 \\
6 \\
7 \\
8 \\
9 \\
10 \\
11 \\
12 \\
13 \\
14 \\
15 \\
16
\end{gathered}
$$

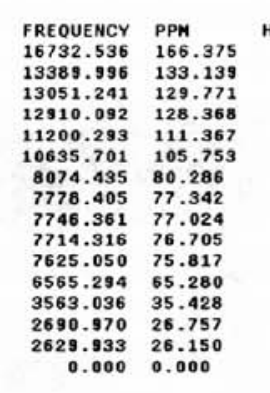

HEIGHT
11.2
59.1
100.6
94.2
25.1
29.2
26.8
146.5
151.2
144.3
39.6
35.0
48.5
42.4
37.5
23.2

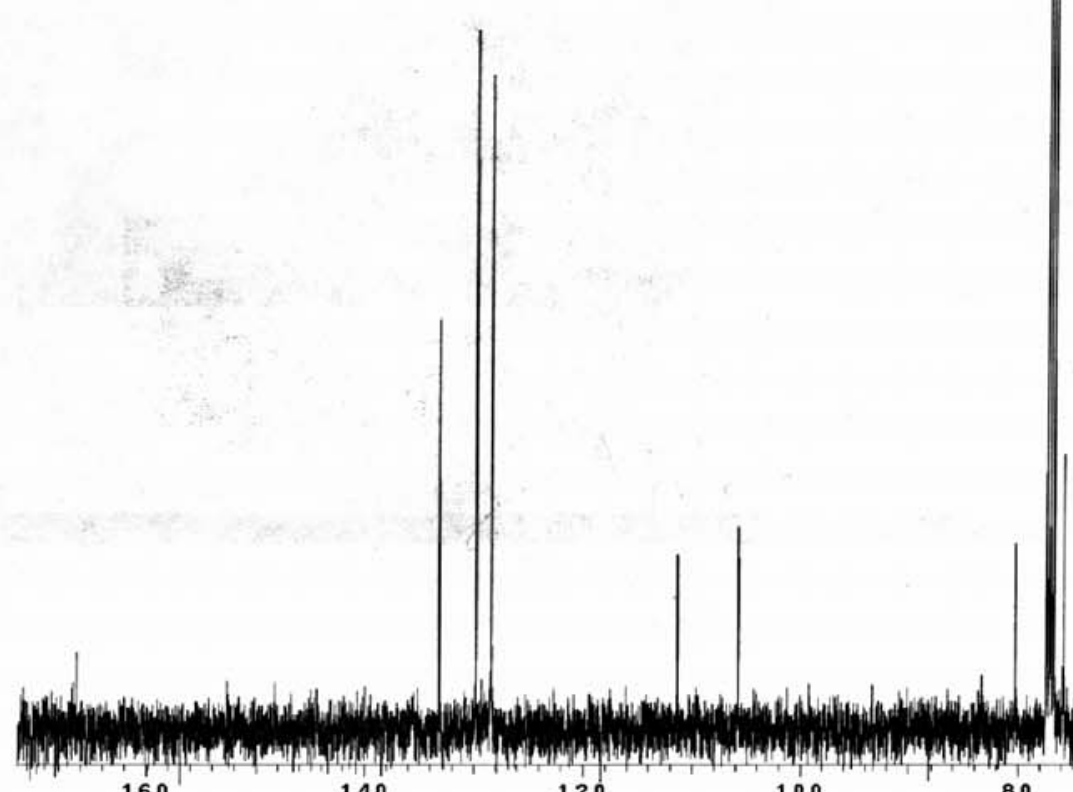

160

140

120

100

80
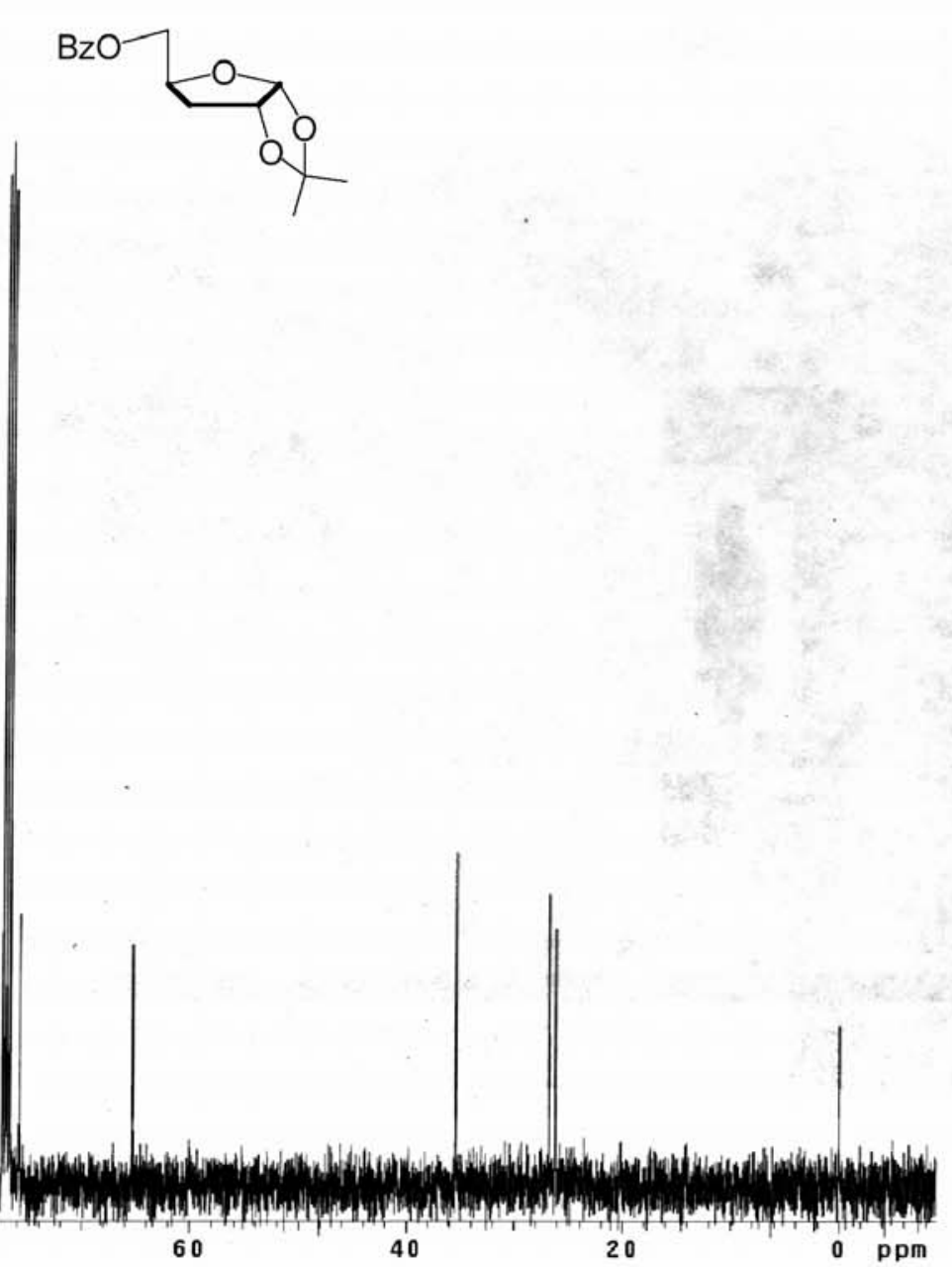
STANDARD 1H OBSERVE

Pulse Sequence: s2pul

Solvent: $\mathrm{CDCl}_{3}$

Ambient temperature

ile: phosphite-admantanol

INOVA-500 "Ni"

PULSE SEQUENCE

Pulse 42.1 degrees

Acq. time $3,333 \mathrm{sec}$

Width $4500.5 \mathrm{~Hz}$

32 repetitions

DATA PROCESSING

Line broadening $0.2 \mathrm{~Hz}$

FT size 32768

Total time $1 \mathrm{~min}, 46 \mathrm{sec}$
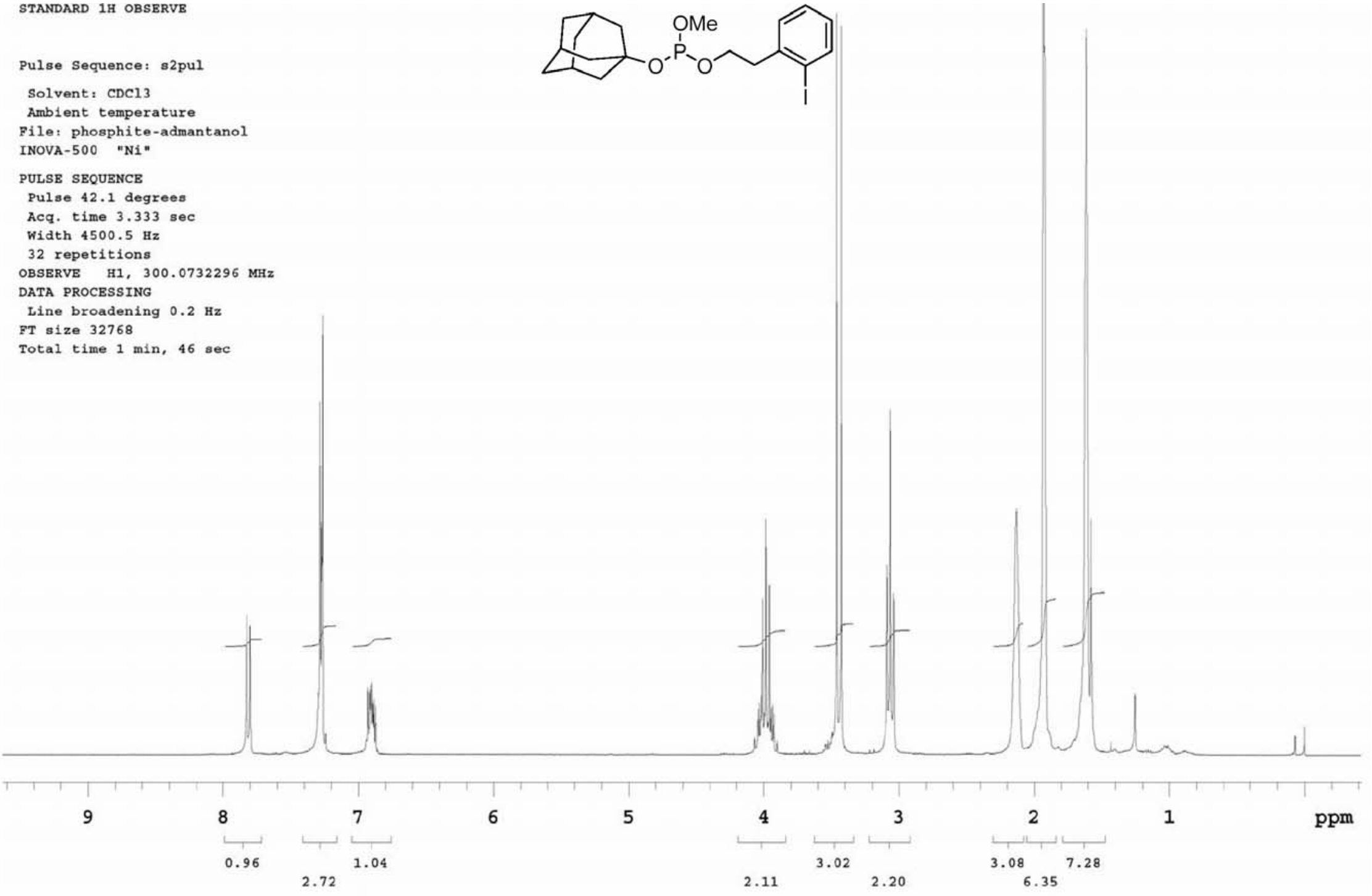
13C OBSERVE

Pulse Sequence: e2pul

Solvent: $\mathrm{CDCl} 3$

Ambient temperature

ile: phosphite-admantano1-c13

INOVA-500 "Ni"

PULSE SEQUENCE

Relax. delay $0.900 \mathrm{se}$

Pulse 45.0 degrees

Acq. time $1.199 \mathrm{sec}$

Width $25000.0 \mathrm{~Hz}$

336 repetitions

OBSERVE C13, 100.5712676 MH

DECOUPLE H1, 399.9669644 MHz

Power $44 \mathrm{~dB}$

continuously on

WALTZ-16 modulated

DATA PROCESSING

Line broadening $1.0 \mathrm{~Hz}$

Total time $70 \mathrm{hr}, 16 \mathrm{~min}, 25 \mathrm{sec}$

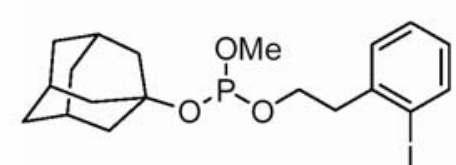

号華

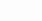

180

160

140

ppm 
STANDARD 1H OBSERVE

Pulse Sequence: s2pul

Solvent: $\mathrm{CDC} 13$

Ambient temperature

File: phosphite-admantanol-p31

INOVA-500 "Ni"

PULSE SEQUENCE

Pulse 45.0 degrees

Acq. time $1.069 \mathrm{sec}$

Width $59880,2 \mathrm{~Hz}$

64 repetitions

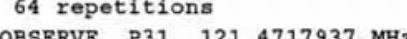

DECOUB $\mathrm{HI}, 300.0746975 \mathrm{MHz}$

Power $45 \mathrm{~dB}$

continuously on

WALTZ-16 mody

DATA PROCESSING

Line broadening $0.5 \mathrm{~Hz}$

FT size 65536

Total time $3 \mathrm{hr}, 35 \mathrm{~min}, 31 \mathrm{sec}$

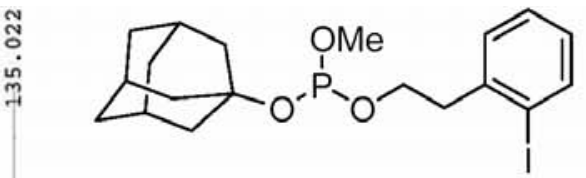

ส

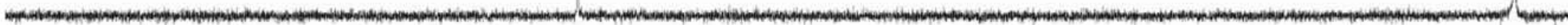


STANDARD 1 H OBSERVE

Pulse Sequence: s2pul

Solvent: $\mathrm{CDCl}_{3}$

Ambient temperature

ile: phosphite-tertiary-steroid

INOVA-500 "Ni"

PULSE SEQUENCE

Pulse 41.4 degrees

Acq. time $2.666 \mathrm{sec}$

width $3630.1 \mathrm{~Hz}$

16 repetitions

399.9649444

BSSIN

total time 0 min, $42 \mathrm{sec}$
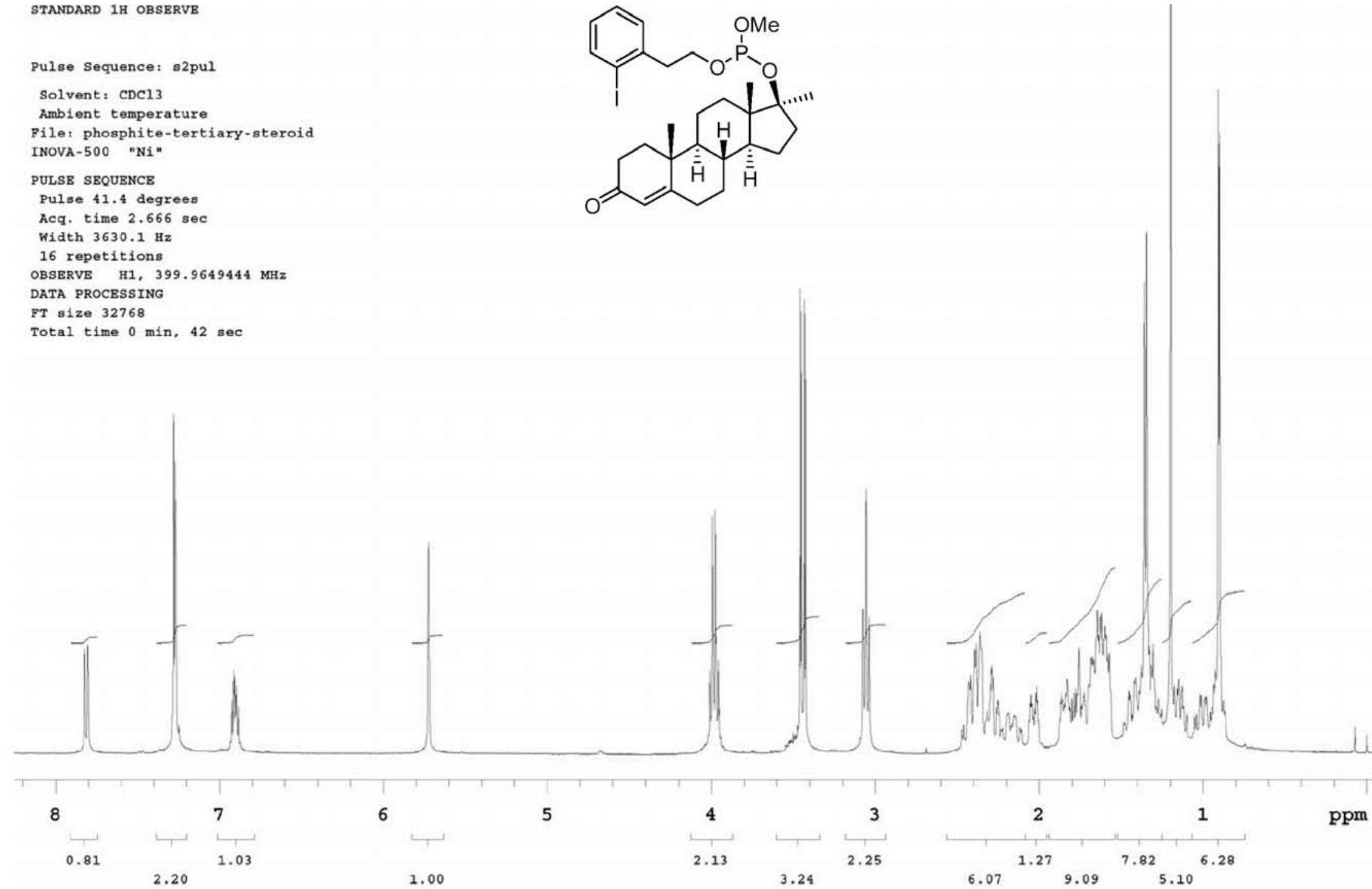
PHOSPHORUS OBSERVE

STANDARD PARAMETERS

PHOSPHATE REGIOS

Pulse Sequence: s2pul

Solvent: $\mathrm{CDC13}$

Ambient temperature

File: phosphite-teriary-steroid-p31

INOVA-500 "N1"

PULSE SBQUENCE

Pulse 44.2 degree

Pulce 44.2 degreses

Acq. time 0.800 sec

Width 50000.0 He

128 repetitions

BSERVE P31, $161.9086874 \mathrm{MHz}$

DECOUPLE H1, $399.9669644 \mathrm{MHz}$

Power $44 \mathrm{~dB}$

continuousiy on

WALTZ-16 modulate

DATA PROCESSING

Line broadening $1.0 \mathrm{~Hz}$

FT size 131072

Total time $1 \mathrm{~min}, 43 \mathrm{sec}$
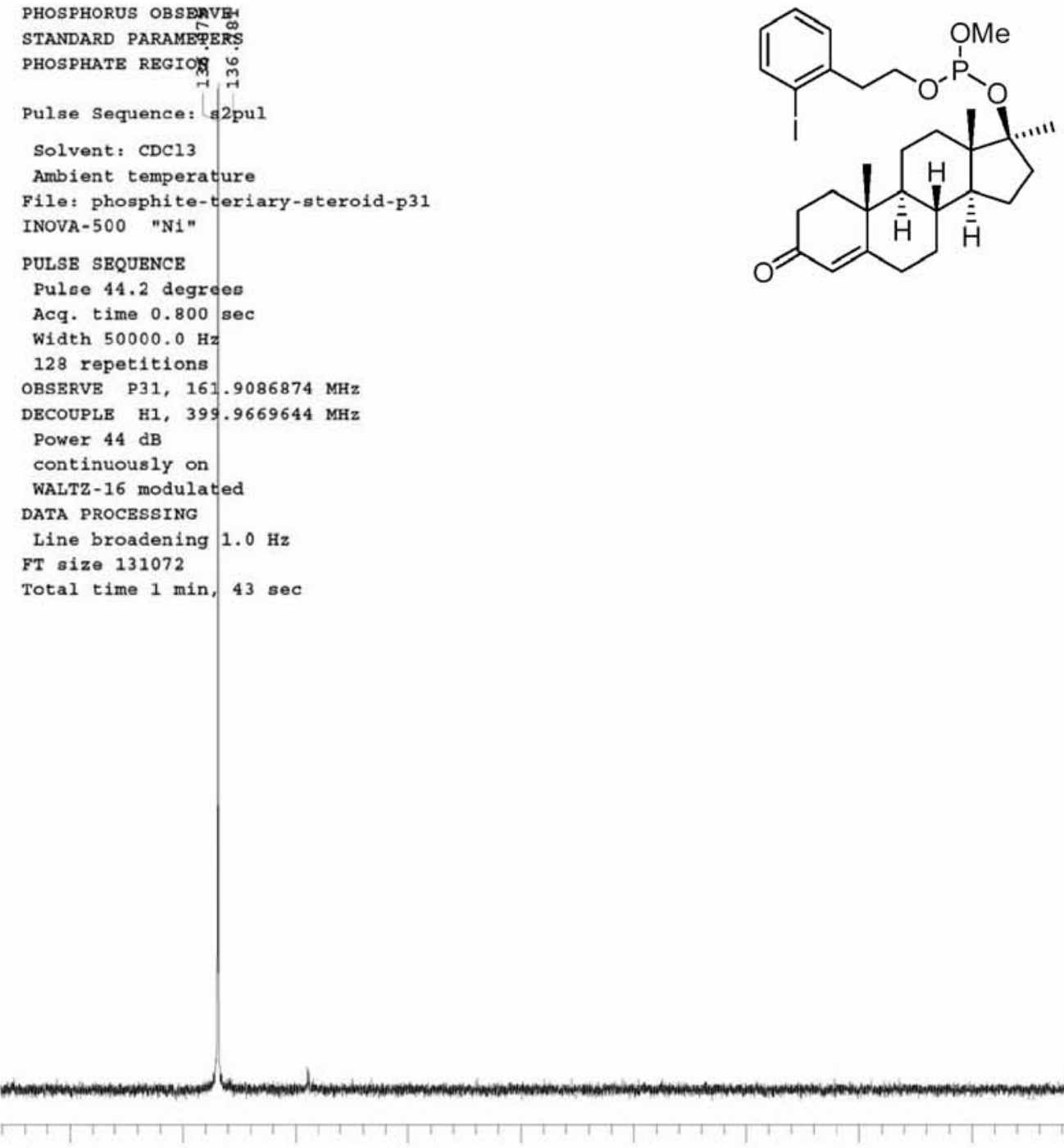

$1+1+1$

120

110

100

90

80

70

60

50

40

30

20

10

ppm 
STANDARD $1 \mathrm{H}$ OBSERVE

Pulse Sequence: s2pul

Solvent: $\mathrm{CDCl} 3$

Ambient temperature

ile: phosphite-tertiary-steroid-reduction

INOVA-500 "Ni"

PULSE SEQUENCE

Pulse 41.4 degrees

Acq. time $2,666 \mathrm{sec}$

wiath 3630,1 H:

16 repetitions

T size 32768

Total time $0 \mathrm{~min}, 42 \mathrm{sec}$

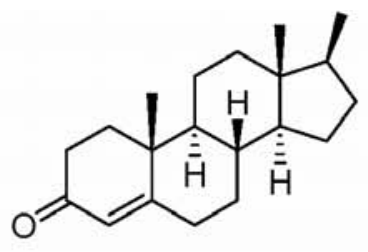

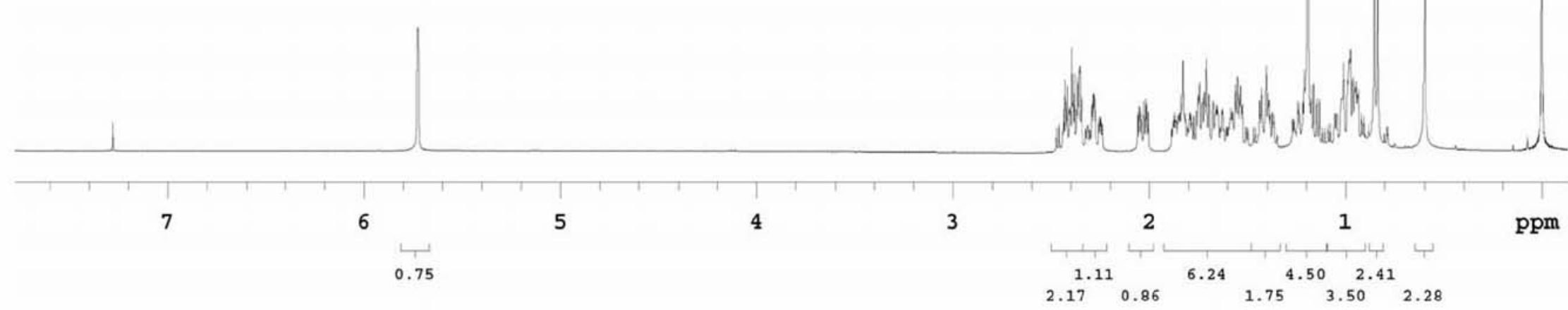




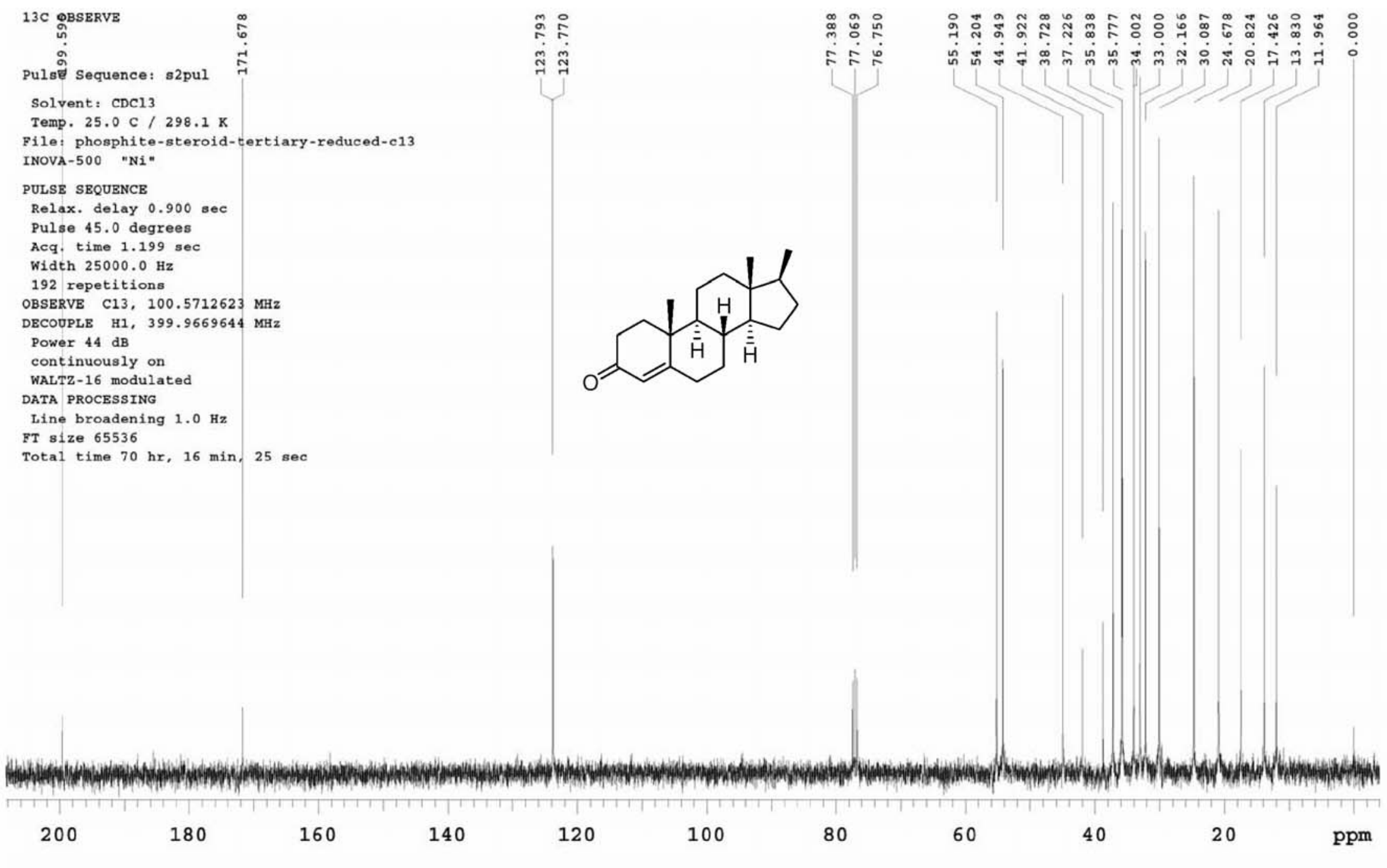

